\title{
MATERIAIS PARA CÁTODOS DE BATERIAS SECUNDÁRIAS DE LÍTIO
}

\author{
Hamilton Varela ", Fritz Huguenin, Marcos Malta e Roberto M. Torresi ${ }^{*}$ \\ Instituto de Química de São Carlos, Universidade de São Paulo, CP 780, 13560-970 São Carlos - SP
}

Recebido em 21/12/00; aceito em 4/7/01

\begin{abstract}
MATERIALS FOR CATHODES OF SECONDARY LITHIUM BATTERIES. In this work, cathodes employed in secondary lithium batteries are reviewed. These cathodes have great technologic and scientific importance, specifically, materials for cathodes as electronic conductor polymers (ECP), transition metal oxides (TMO) and nanocomposites of ECP/TMO. The use of a specific cathodic material is based in some intrinsic characteristics that improve the performance of the battery. Thus, some vantages and disvantages of these insertion compounds are discussed, as lithium insertion capacity, energy density, and the ciclability of these materials.
\end{abstract}

Keywords: secondary lithium batteries; cathodic materials; transition metal oxides; electronic conducting polymers; nanocomposites.

\section{INTRODUÇÃO}

Uma fonte eletroquímica de potência ou bateria pode ser definida como um dispositivo capaz de converter diretamente a energia liberada numa reação química em energia elétrica. As baterias apresentam basicamente duas funções principais, a primeira é agir como fontes portáteis de potência elétrica. A segunda, que tem crescido em importância nos últimos trinta anos, está baseada na habilidade de certos sistemas eletroquímicos de armazenar a energia suprida por uma fonte externa. Tais baterias são utilizadas, em veículos elétricos, fontes de emergência, como parte de um sistema de fornecimento de curta duração para demandas em pico e em conjunção com fontes renováveis de energia, como solar ou eólica, por exemplo.

A primeira descrição de uma bateria eletroquímica foi feita pelo italiano Alessandro Volta em 1800. Tal "descoberta" representa o marco na história da eletroquímica e, particularmente, na história dos dispositivos denominados genericamente baterias. Desde então, um notável progresso tem sido feito na área de armazenamento eletroquímico de energia. Hoje, pode-se enumerar uma grande variedade de dispositivos englobados na categoria de baterias: células metal-ar, metal-hidreto metálico (Ni-HM), níquel-cádmio (Ni-Cd), células térmicas, íons-Lítio, entre outras.

O mercado para dispositivos primários consiste basicamente na produção de baterias para aparelhos portáteis. As baterias secundárias ou recarregáveis representam maior interesse devido à grande demanda atual de aparelhos celulares e microcomputadores portáteis. Em 1997, por exemplo, dos 34 bilhões de dólares movimentados com baterias, 24 foram destinados às baterias secundárias ${ }^{1}$.

Graças à simplicidade e reversibilidade das reações eletroquímicas de inserção ou intercalação, a grande maioria dos dispositivos secundários que operam à temperatura ambiente é baseada nos chamados eletrodos de inserção. Como exemplos, têm-se os dispositivos secundários aquosos, como hidreto metálico/ $\mathrm{NiOOH}$ ou $\mathrm{Zn} / \mathrm{MnO}_{2}$, que envolvem a inserção de $\mathrm{H}^{+}$no hidreto metálico ${ }^{2,3}$ ou $\mathrm{MnO}_{2}^{4,5}$. Até mesmo o mecanismo de redução do eletrodo positivo das baterias de chumbo ácido, $\mathrm{PbO}_{2}^{6,7}$ pode ser atribuído ao processo de inserção de $\mathrm{H}^{+8}$.

\footnotetext{
\# endereço atual: Fritz-Haber-Institut der Max-Planck-Gesellschaft, Faradayweg 4-6, D-14195 Berlin, Germany.

* e-mail: torresi@iqsc.sc.usp.br
}

Considerando-se a utilização de cátions metálicos no processo de intercalação em sistemas não aquosos, nenhuma espécie pode penetrar tão facilmente em sólidos hospedeiros através de reações de inserção como os cátions $\mathrm{Li}^{+}$. Assim, não surpreendentemente, entre os mais estudados dispositivos secundários de alta densidade de energia estão os que utilizam o lítio. Aspectos científicos relacionados aos eletrodos utilizados em baterias secundárias de lítio foram recentemente discutidos em uma extensa revisão ${ }^{8}$.

O objetivo do presente artigo de revisão é discutir o estado da arte dos materiais catódicos ulizados em dispositivos secundários de lítio. A abordagem segue uma distribuição sistemática dos materiais catódicos mais comuns em classes, nas quais são discutidas as propriedades fundamentais frente à sua aplicação específica. As classes apresentadas são: óxidos de metais de transição (OMT), polímeros condutores eletrônicos (PCEs) e eletrodos sólidos de polimerização redox (ESPR), além dos materiais nanoestruturados e compósitos formados entre materiais das diferentes classes apresentadas. Mesmo considerando-se apenas os eletrodos positivos, em detrimento à tentativa de cobrir todos os materiais, procurou-se enfatizar somente os mais extensivamente estudados. Classes como a dos hospedeiros unidimensionais que utilizam metais de transição e tricalcogenetos (como $\mathrm{TiS}_{3}$ e $\mathrm{NbS}_{3}$ ), por exemplo, não são apresentadas e leitores interessados são remetidos à literatura disponível ${ }^{8-11}$. Por último, ao lado das considerações finais serão discutidas algumas perspectivas na utilização dos materiais catódicos empregados em dispositivos práticos.

\section{BATERIAS SECUNDÁRIAS DE LÍTIO}

Para o desenvolvimento efetivo de uma bateria de alta densidade de energia, faz-se necessária a utilização de materiais eletródicos de alta capacidade de carga. Metais alcalinos são escolhas óbvias nesse sentido, e a maioria das baterias produzidas atualmente é baseada nesses metais como eletrodos negativos. De todos os candidatos a materiais anódicos, o lítio é um dos mais atrativos, já que combina um potencial termodinâmico de eletrodo favorável com uma capacidade específica muito alta, 3,86 $\mathrm{A} \mathrm{h} \mathrm{g}^{-1}$ ou 7,23 $\mathrm{A} \mathrm{h} \mathrm{cm}^{-3}$, além do baixo custo e disponibilidade. Como resultado da sua natureza eletropositiva, o lítio reduz rapidamente a água, e células com ânodo de lítio geralmente utilizam eletrólitos não aquosos, como carbonato de propileno $^{12}$ e metil-propil carbonato ${ }^{13}$, entre outros ${ }^{14-16}$. Além do 
mais, a grande disponibilidade de eletrodos de inserção capazes de trocar quantidades substanciais de íons lítio com cinética relativamente rápida, tem promovido o desenvolvimento de vários tipos de baterias recarregáveis de lítio com diferentes modelos, tamanhos, potências e capacidades. Todas essas baterias utilizam as características superiores do lítio:

(a) Alta voltagem (aproximadamente $3 \mathrm{~V}$ );

(b) Alta densidade de energia;

(c) Baixa taxa de auto-descarga;

(d) Larga faixa de temperatura de operação.

No início dos anos 70 descobriu-se que compostos de intercalação poderiam ser utilizados como eletrodos em dispositivos secundários de lítio. Um composto de intercalação pode ser definido como um sólido capaz de incorporar reversivelmente átomos ou moléculas dentro da sua estrutura (cristalina ou não) sem sofrer grandes variações estruturais ${ }^{17}$. Durante os anos 70 e 80, praticamente todos os trabalhos em eletrodos para baterias secundárias de lítio estavam centrados na utilização de compostos de intercalação como cátodos e lítio metálico (ou em forma de liga) como ânodo. No entanto, observou-se um certo desinteresse por essa configuração em virtude dos problemas relacionados ao ânodo metálico. Quando o lítio é eletroquimicamente depositado no ânodo de lítio durante o recarregamento do dispositivo, esse depósito é mais poroso que o metal original. Esse fenômeno causa problemas como corrosão e formação de dendritos (com conseqüente perda de contato elétrico), que diminuem a vida útil do dispositivo ${ }^{18}$. Com o decorrer dos ciclos de carga/descarga, observa-se uma perda de lítio na interface ânodo/ eletrólito, a qual se torna termodinamicamente instável e permanece cineticamente estável apenas através da presença do filme passivante formado na interface ${ }^{19}$. Apesar de menos pronunciado, esse efeito é ainda presente quando da utilização de ligas contendo lítio.

Em consequiência do que foi exposto, o principal problema dos dispositivos secundários contendo lítio metálico como ânodo pode ser discutido através do aumento de área da interface eletrodo/eletrólito no decorrer dos ciclos de carga/descarga. Como essa interface não é termodinamicamente estável, a célula vai se tornando mais sensível às variáveis térmicas, mecânicas e elétricas. Esse problema pode ser contornado através da utilização de dispositivos denominados íon-Li. Nesse caso, são utilizados dois eletrodos de intercalação, altamente reversíveis, onde a área superficial do ânodo em dispositivos práticos é de aproximadamante $1 \mathrm{~m}^{2} \mathrm{~g}^{-1} \mathrm{e}$, aparentemente, não aumenta com os $\operatorname{ciclos}^{20}$. Isto significa que a segurança e o desempenho da célula podem ser considerados independentes do número de ciclos.

Materiais anódicos como carbono e grafite são mais freqüentemente utilizados e perfeitamente aceitáveis em aplicações práticas devido às propriedades como custo, disponibilidade, desempenho e potencial versus lítio ${ }^{17}$. Alternativamente aos materiais carbonáceos anódicos, a utilização de ligas metálicas do tipo $\mathrm{Li}_{x} \mathrm{M}(\operatorname{com~} \mathrm{M}=\mathrm{Mg}$, $\mathrm{Si}, \mathrm{Ge}, \mathrm{Sn}, \mathrm{Pb}, \mathrm{Bi}, \mathrm{Pt}, \mathrm{Ag}$, etc.) vêm sendo extensivamente estudadas, com resultados superiores aos ânodos de grafite litiado, além da vantagem adicional de ausência da co-intercalação de solvente ${ }^{21}$. Entretanto, devido à menor capacidade de carga, e pior performance durante os ciclos de carregamento/descarregamento, esses materiais vêm sendo preteridos aos materiais carbonáceos anódicos ${ }^{8}$.

O sistema íon-Li introduzido por Scrosati et al. ${ }^{22} \mathrm{e} \mathrm{Armand}^{23}$ conjuntamente, e produzido pela Sony em $1991^{24}$, tem experimentado um crescimento espetacular desde então. Este dispositivo é também denominado sistema secundário rocking chair ou swing, já que as reações da célula essencialmente transportam íons lítio de um eletrodo para outro de forma reversível ${ }^{25-27}$.

A Tabela $1^{28}$ ilustra os mais recentes avanços em baterias secundárias íon-Li. Exceto para os dispositivos utilizando cátodos de $\mathrm{LiMn}_{2} \mathrm{O}_{4}$, todos os outros sistemas tiveram sua produção iniciada em 1999. O dispositivo produzido pela Moltech utilizando cátodo polimérico é o único disponível comercialmente na atualidade. No entanto, informações a respeito do material catódico não são disponíveis, tratando-se provavelmente de um compósito entre polímero e 2,5 - dimercapto 1,3,4 - tiadiazol (DMcT), como será discutido posteriormente.

De forma geral, o desempenho de um dispositivo secundário de íon-Li é limitado principalmente pelo material catódico e eletrólito, tendo em vista as propriedades do lítio como eletrodo negativo. De fato, segundo Broussely et al. ${ }^{29}$, os valores médios de capacidade a serem considerados em um dispositivo estão em torno de 140 e 350 $\mathrm{A} \mathrm{h} \mathrm{kg}^{-1}$, para o cátodo e o ânodo, respectivamente.

\section{MATERIAIS CATÓDICOS}

\section{Materiais Inorgânicos: Óxidos de Metais de Transição (OMT)}

Como resultado da intensa pesquisa em materiais catódicos de alta energia, os óxidos de metais de transição tornaram-se alguns dos mais promissores, considerando-se a aplicação comercial em baterias de lítio. Esses compostos dispõem de muitos atributos favoráveis que incluem: boa capacidade, alta energia específica, baixa taxa de autodescarga e excelente ciclo de vida. Entre os principais materiais que vêm sendo estudados recentemente, pode-se citar os óxidos litiados de níquel $\left(\mathrm{LiNiO}_{2}\right)$, cobalto $\left(\mathrm{LiCoO}_{2}\right)$, manganês $\left(\mathrm{LiMn}_{2} \mathrm{O}_{4}\right)$ e vanádio $\left(\mathrm{LiV}_{2} \mathrm{O}_{5}\right)$. Os cátodos resultantes são denominados condutores mistos, devido às conduções eletrônica e iônica operantes. Uma vez que materiais policristalinos são usados em aplicações práticas, aspectos como relações entre estrutura, microestrutura, transporte de massa e carga devem ser levados em conta na otimização das suas propriedades, quando do seu uso como cátodos ${ }^{30}$.

A eletro-intercalação em óxidos de metais de transição está associada ao fato de que quando esses sistemas são oxidados ou reduzidos eletroquimicamente, a ligação oxigênio/metal é mantida (graças à sua alta energia) e o processo de eletroneutralização deve ser efetuado pela intercalação de cátions na rede hospedeira. Nas baterias recarregáveis, é desejável que os íons $\mathrm{Li}^{+}$sejam inseridos intersticialmente dentro da estrutura hospedeira durante a descarga e, posteriormente, expulsos durante a recarga com pequena ou nenhuma modificação estrutural do material hospedeiro. Em termos de energia, o desempenho do sistema é limitado pela velocidade com a qual

Tabela 1. Características de alguns dispositivos secundários de lítio disponíveis comercialmente ${ }^{28}$

\begin{tabular}{|c|c|c|c|c|c|}
\hline Companhia & $\begin{array}{c}\text { Configuração } \\
\text { Cátodo/Eletrólito/Ânodo }\end{array}$ & $\begin{array}{l}\text { Voltagem } \\
\qquad / \mathrm{V}\end{array}$ & $\begin{array}{l}\text { Tamanho } \\
/ \mathrm{mm}\end{array}$ & $\begin{array}{l}\text { Capacidade } \\
\text { /mA h }\end{array}$ & $\begin{array}{l}\text { D.E. } \\
/ \mathrm{W} \mathrm{h} \mathrm{kg}^{-1}\end{array}$ \\
\hline Ultralife & $\mathrm{LiMn}_{2} \mathrm{O}_{4} / \mathrm{PVDF}($ gel $) /$ Grafite & 3,7 & $51 \times 103 \times 6,5$ & 1.700 & 105 \\
\hline Valence & $\mathrm{LiMn}_{2} \mathrm{O}_{4}^{4} / \mathrm{PVDF}(\mathrm{gel}) /$ Grafite & 3,7 & $102 \times 127 \times 5$ & 3.900 & 110 \\
\hline Sony & $\mathrm{LiCoO}_{2} / \mathrm{PVDF}(\mathrm{gel}) /$ Grafite & 3,7 & $35 \times 62 \times 3,8$ & 540 & 125 \\
\hline Electrofuel & $\mathrm{LiCoO}_{2} / \mathrm{PVDF}($ gel $) /$ Grafite & 3,6 & $108 \times 138 \times 6$ & 10800 & 175 \\
\hline Moltech & Polímero (???)/Solvente orgânico/Metal Lítio & 2,1 & $34 \times 48 \times 5$ & 800 & 170 \\
\hline
\end{tabular}


o lítio pode ser inserido ou expulso dos compostos de inserção. Em linhas gerais, assume-se que o processo de intercalação envolve três etapas principais ${ }^{31}$ :

(a) difusão e migração dos íons solvatados no eletrólito;

(b) desolvatação e injeção dos íons $\mathrm{Li}^{+}$dentro da vacância estrutural;

(c) difusão dos íons $\mathrm{Li}^{+}$dentro da rede hospedeira.

Um aspecto importante é que, devido a capacidade de fornecer $\mathrm{Li}^{+}$, os óxidos litiados se comportam como um eletrodo fornecedor de lítio e, portanto, podem ser convenientemente acoplados a um eletrodo de carbono para formar uma bateria do tipo $\mathrm{C} / \mathrm{LiMO}_{2}(\mathrm{M}=\mathrm{Co}, \mathrm{Ni})$ em sua forma completamente descarregada ${ }^{32}$. A ativação dessa bateria requer uma etapa de carregamento envolvendo a remoção de íons lítio do eletrodo de $\mathrm{LiMO}_{2}$ e sua inserção no eletrodo de carbono, de acordo com um processo reversível rocking-chair do tipo ${ }^{30}$ :

$$
\mathrm{LiMO}_{2}+6 \mathrm{C} \leftrightarrow \mathrm{Li}_{(1-x)} \mathrm{MO}_{2}+\mathrm{Li}_{\mathrm{x}} \mathrm{C}_{6}
$$

Os compostos de $\mathrm{LiMO}_{2}$ são normalmente obtidos por reações em alta temperatura a partir de quantidades estequiométricas de hidróxido de lítio e dos óxidos metálicos selecionados. Esses óxidos possuem uma estrutura baseada em uma rede de empacotamento fechado de átomos de oxigênio com os íons de $\mathrm{Li}^{+}$e $\mathrm{M}^{3+}$ ordenados em planos (111) alternados em uma estrutura cúbica conhecida como rock-salt ${ }^{33}$. Portanto, essas estruturas lamelares fornecem um espaço intersticial bidimensional, o qual permite a fácil expulsão dos íons lítio $^{34}$. Os estudos iniciais realizados por Plichta et al. ${ }^{35} \mathrm{com}$ relação à reversibilidade do $\mathrm{Li}_{\mathrm{x}} \mathrm{CoO}_{2}$, mostraram que entre as regiões $0,3 \leq \mathrm{x}$ $\leq 1$, existem 3 transições de fases reversíveis. Também foi constatada a existência de uma quarta fase (irreversível, para voltagens superiores a 4,3 V versus $\mathrm{Li} / \mathrm{Li}^{+}$) para $\mathrm{x}<0,3$, onde as camadas da estrutura de $\mathrm{Li}_{\mathrm{x}} \mathrm{CoO}_{2}$ são destruídas.

Atualmente, buscam-se novas rotas de síntese para os óxidos de $\mathrm{Li}_{\mathrm{x}} \mathrm{MO}_{2}$ em temperaturas menos drásticas e que não afetem as propriedades eletroquímicas. A obtenção de $\mathrm{LiCoO}_{2}$ pelo método solgel já foi descrita na literatura ${ }^{36}$. Uma outra rota de síntese é a reação de troca catiônica (utilizando soluções aquosas de $\mathrm{LiOH}_{2} \mathrm{H}_{2} \mathrm{O}$ contendo $\mathrm{H}_{x} \mathrm{MO}_{2}$ ) realizada em condições hidrotérmicas. Os produtos obtidos exibem desempenho eletroquímico comparáveis às fases produzidas em alta temperatura ${ }^{37}$.

Outro material que vem sendo amplamente estudado é o $\mathrm{LiNiO}_{2}$, cujo custo de produção é consideravelmente menor que o $\mathrm{LiCoO}_{2}{ }^{38}$. Entretanto, a síntese estequiométrica desse material é um tanto complexa, e seu comportamento eletroquímico é fortemente dependente da existência de defeitos estruturais formados durante este processo $^{39-41}$. Além disso, sob extensiva deintercalação (na qual x se aproxima de zero e o estado de valência do níquel se torna próximo de 4+) os íons níquel migram de seus sítios para as vacâncias deixadas pelos íons lítio. Tal migração cria várias limitações difusionais na reinserção de $\mathrm{Li}$, reduzindo portanto, o desempenho do dispositi$\mathrm{vo}^{42}$. Um caminho conveniente para superar essa dificuldade pode ser o uso de fases mistas de composição $\mathrm{LiNi}_{1-\mathrm{y}} \mathrm{Co}_{\mathrm{y}} \mathrm{O}_{2}^{43-45}$ ou $\mathrm{LiNi}_{\mathrm{x}} \mathrm{Mn}_{2-\mathrm{x}} \mathrm{O}_{4}^{46,47}$, por exemplo.
Apesar das altas voltagens e elevada energia específica das células constituídas de $\mathrm{LiCoO}_{2}$ e $\mathrm{LiNiO}_{2}$, há grande interesse em substituir esses materiais catódicos por outros mais baratos, mas com semelhante desempenho eletroquímico. Um dos óxidos com grande potencial para tal é o espinélio que contém íons manganês, $\mathrm{Li}_{x} \mathrm{Mn}_{2} \mathrm{O}_{4}^{48,49}$. $\mathrm{O} \mathrm{Li}_{x} \mathrm{Mn}_{2} \mathrm{O}_{4}$ opera com um potencial de aproximadamente $4 \mathrm{~V}$ versus $\mathrm{Li} / \mathrm{Li}^{+}$no intervalo entre $0<\mathrm{x} \leq 1$ e tem uma capacidade específica teórica de $154 \mathrm{~A} \mathrm{~h} \mathrm{~kg}^{-1}$, baseado na massa do eletrodo completamente oxidado $\left(\mathrm{MnO}_{2}\right)^{50}$. Esse material é normalmente sintetizado pela reação no estado sólido de óxidos ou sais de lítio e manganês a $750{ }^{\circ} \mathrm{C}$ aproximadamente ${ }^{51}$. Nessas condições de reação, o produto possui uma estrutura de espinélio com a notação padrão $\mathrm{Li}_{8 \mathrm{a}}\left[\mathrm{Mn}_{2}\right]_{16 \mathrm{~d}} \mathrm{O}_{4}$, no qual os íons lítio estão localizados nos sítios tetraédricos $8 \mathrm{a}$ e os íons manganês nos sítios octaédricos $16 \mathrm{~d}$ (grupo espacial Fd3m) $)^{52}$. Esse composto exibe dois platôs nas curvas de potencial versus $\mathrm{Li} / \mathrm{Li}^{+}$: um a $4 \mathrm{~V}$, correspondendo à extração de lítio dos sítios tetraédricos $8 \mathrm{a}$, e outro a $3 \mathrm{~V}$, referente à inserção de um lítio adicional nos sítios octaédricos vazios 16c. Enquanto a inserção/extração de lítio na região de $4 \mathrm{~V}$ mantém a simetria cúbica do espinélio, na região de $3 \mathrm{~V}$ ocorre uma transição de fase envolvendo o $\mathrm{LiMn}_{2} \mathrm{O}_{4}$ cúbico e o $\mathrm{Li}_{2} \mathrm{Mn}_{2} \mathrm{O}_{4}$ tetragonal. A perda de capacidade na região de $3 \mathrm{~V}$ limita o uso do material somente à região em torno de $4 \mathrm{~V}^{53}$.

$\mathrm{O}$ espinélio de $\mathrm{LiMn}_{2} \mathrm{O}_{4}$ exibe uma menor capacidade prática que o $\mathrm{LiCoO}_{2}{ }^{54}$, além de uma considerável perda de capacidade em temperaturas acima de $50{ }^{\circ} \mathrm{C}^{55,56}$. Acredita-se que a instabilidade química da estrutura do espinélio em eletrólitos contendo lítio resulta em uma dissolução do Mn, sendo a origem do pobre desempenho observado em altas temperaturas ${ }^{57}$. Uma outra dificuldade encontrada para aplicação prática é a possiblidade do $\mathrm{LiMn}_{2} \mathrm{O}_{4}$ exibir uma perda de capacidade significativa durante a ciclagem. Em adição à instabilidade em relação ao eletrólito orgânico (com possível dissolução do óxido) $)^{58}$, outras causas da perda de capacidade têm sido sugeridas, incluindo o início do efeito Jahn-Teller nos eletrodos de $\mathrm{Li}_{\mathrm{x}} \mathrm{Mn}_{2} \mathrm{O}_{4}$ descarregados $(\mathrm{x} \sim 1)^{59}$, e a estrutura local não homogênea do $\mathrm{Li}_{\mathrm{x}} \mathrm{Mn}_{2} \mathrm{O}_{4}{ }^{60}$, por exemplo.

A Tabela 2 sumariza as reações operantes nos cátodos utilizando $\mathrm{LiMn}_{2} \mathrm{O}_{4}, \mathrm{LiCoO}_{2}, \mathrm{LiNiO}_{2}$ e $\mathrm{LiNi}_{0.8} \mathrm{Co}_{0,2} \mathrm{O}_{2}$. Ao lado das reações teóricas (ou seja, envolvendo o carregamento total) são apresentadas as prováveis reações operantes observadas em carregamento prático com os valores descritos na literatura ${ }^{61-63}$. Através da razão entre as cargas observada e teórica $\left(\mathrm{C}_{\mathrm{o}} / \mathrm{C}_{\mathrm{t}}\right)$ é possível comparar melhor os dispositivos. Apesar da capacidade obtida ser consideravelmente menor que nos outros casos mostrados, o melhor desempenho entre os cátodos ilustrados pode ser atribuído ao formado por $\mathrm{LiMn}_{2} \mathrm{O}_{4}$, no qual a capacidade atingida foi da ordem de $81 \%$ do valor teórico, qualificando dessa maneira o $\mathrm{LiMn}_{2} \mathrm{O}_{4}$ para futuras investigações ${ }^{64-66}$. De fato, essa razão diminui um pouco quando o $\mathrm{LiMn}_{2} \mathrm{O}_{4}$ é utilizado na presença de eletrólitos orgânicos, devido à menor faixa de potencial utilizada, e atinge em torno de $75 \%{ }^{56}$, nesse sentido, a adição de cobalto tem sido empregada com sucesso no aumento da capacidade de tais dispositivos ${ }^{67,68}$. Armstrong et al. ${ }^{60}$ reportaram recentemente capacidades da ordem de $200 \mathrm{~A} \mathrm{~h} \mathrm{~kg}^{-1}$ para o $\mathrm{LiMn}_{0,9} \mathrm{Co}_{0,1} \mathrm{O}_{2}$, graças

Tabela 2. Alguns óxidos de metais de transição, com as reações globais, capacidade de carga teórica e experimental ${ }^{61-63}$

\begin{tabular}{|c|c|c|c|c|c|}
\hline Cátodo & Carregamento Total & Carregamento observado & $\mathrm{C}_{\mathrm{t}}{ }^{*}$ & $\mathrm{C}_{\mathrm{o}} * *$ & $\mathrm{C}_{\mathrm{o}} / \mathrm{C}_{\mathrm{t}}$ \\
\hline $\mathrm{LiMn}_{2} \mathrm{O}_{4}$ & $\mathrm{LiMn}_{2} \mathrm{O}_{4} \rightarrow \mathrm{Li}+\mathrm{Mn}_{2} \mathrm{O}_{4}$ & $\mathrm{LiMn}_{2} \mathrm{O}_{4} \rightarrow 0,74 \mathrm{Li}+\mathrm{Li}_{0,26} \mathrm{Mn}_{2} \mathrm{O}_{4}$ & 148 & 120 & 0,81 \\
\hline $\mathrm{LiCoO}_{2}$ & $\mathrm{LiCoO}_{2}^{4} \rightarrow \mathrm{Li}+\mathrm{CoO}_{2}^{4}$ & $\mathrm{LiCoO}_{2} \rightarrow 0,58 \mathrm{Li}+\mathrm{Li}_{0,42} \mathrm{CoO}_{2}^{4}$ & 274 & 142 & 0,52 \\
\hline $\mathrm{LiNiO}_{2}$ & $\mathrm{LiNiO}_{2} \rightarrow \mathrm{Li}+\mathrm{NiO}_{2}$ & $\mathrm{LiNiO}_{2} \rightarrow 0,53 \mathrm{Li}+\mathrm{Li}_{0,47}^{,+42} \mathrm{NiO}_{2}^{2}$ & 275 & 145 & 0,53 \\
\hline $\mathrm{LiNi}_{0,8} \mathrm{Co}_{0,2} \mathrm{O}_{2}$ & $\mathrm{LiNi}_{0,8} \mathrm{Co}_{0,2} \mathrm{O}_{2} \rightarrow \mathrm{Li}+\mathrm{Ni}_{0,8} \mathrm{Co}_{0,2} \mathrm{O}_{2}$ & $\mathrm{LiNi}_{0,8} \mathrm{Co}_{0,2} \mathrm{O}_{2} \rightarrow 0,66 \mathrm{Li}+\mathrm{Li}_{0,34} \mathrm{Ni}_{0,8} \mathrm{Co}_{0,2} \mathrm{O}_{2}$ & 274 & 180 & 0,66 \\
\hline
\end{tabular}

${ }^{*}$ Capacidade teórica, $\mathrm{A} \mathrm{h} \mathrm{kg}^{-1}$; ${ }^{* *}$ Capacidade observada, $\mathrm{A} \mathrm{h} \mathrm{kg}^{-1}$ 
à supressão do efeito Jahn-Teller pela substituição de $10 \%$ do Mn pelo Co.

A Figura 1 ilustra a variação de potencial $v s$. Li em função da composição de $\mathrm{Li}(0<\mathrm{x}<1)$ inserido na estrutura de $\mathrm{Li}_{x} \mathrm{CoO}_{2}, \mathrm{Li}_{x} \mathrm{NiO}_{2}$ e $\mathrm{Li}_{\mathrm{x}} \mathrm{Mn}_{2} \mathrm{O}_{4}$ a baixas velocidades de carga e descarga ${ }^{69}$. Estes altos valores de potencial justificam a denominação dada a esses materiais, conhecidos como cátodos de alta voltagem ou eletrodos de 4 volts, os quais são bastante favoráveis para a aplicação prática dessas baterias de lítio em razão das suas elevadas densidades de energia. Os diferentes comportamentos das curvas mostradas na figura são atribuídos tanto a fatores entálpicos como entrópicos do sistema. A variação de entalpia durante a intercalação dos íons $\mathrm{Li}^{+}$está normalmente associada às variações do potencial químico dos elétrons, às interações entre os íons $\mathrm{Li}^{+}$localizados em sítios vizinhos e às mudanças na microestrutura do filme. Enquanto a variação de entropia pode estar relacionada, por exemplo, à formação de duas fases ou destruição da estrutura, ou seja, uma reorganização estrutural mais significativa $^{70}$.

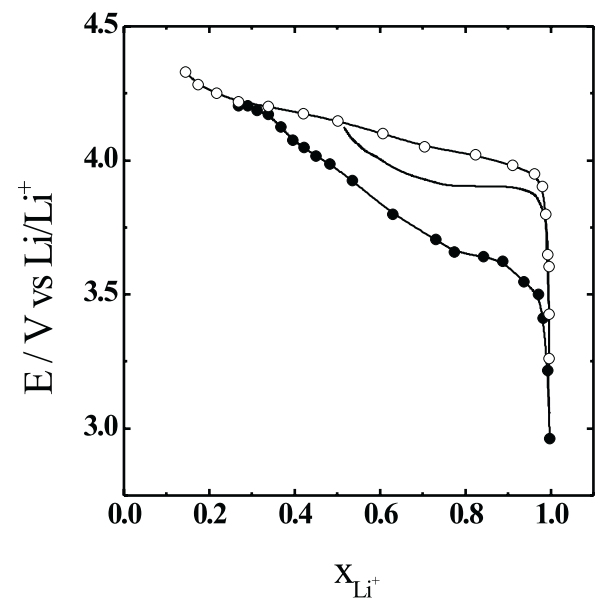

Figura 1. Variação do potencial em função da intercalação de $\mathrm{Li}^{+}$nos filmes

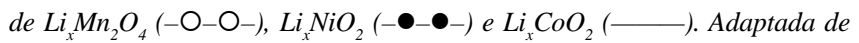
ref. [69]

Vários grupos de pesquisa no Brasil e no exterior têm-se dedicado ao estudo do óxido de vanádio em decorrência de sua versatilidade em aplicações na área de dispositivos eletrocrômicos, catálise e baterias recarregáveis ${ }^{71-78}$. Os cátodos de baterias de lítio constituídos de pentóxido de vanádio foram provavelmente os mais estudados em razão de suas propriedades eletroquímicas, dispondo de uma alta capacidade específica e densidade de energia.

$\mathrm{O}_{2} \mathrm{O}_{5}$ pode ser obtido pelo processo sol-gel através da acidificação do metavanadato ou pela hidrólise e condensação de alcóxidos de vanádio. Alcóxidos metálicos, $\mathrm{VO}(\mathrm{OR})_{3}(\mathrm{R}=\mathrm{Et}, \mathrm{n}-$ But, n-Prop, iso-Prop, t-Am), são precursores moleculares versáteis para síntese sol-gel de óxidos metálicos em razão dos grupos eletronegativos OR, tornando-os bastantes susceptíveis a ataques nucleofílicos. Desta forma, esses alcóxidos são extremamente reativos frente à água, devido à alta eletrofilicidade do vanádio, formando hidróxidos e, posteriormente, óxidos hidratados em conseqüência do processo de condensação ${ }^{79}$.

O controle das condições de síntese desses óxidos pode ser feito eletroquimicamente, gerando produtos com características pré-determinadas. Cristais de óxido de vanádio $\left(\mathrm{c}-\mathrm{V}_{2} \mathrm{O}_{5}\right)$ usados como materiais catódicos exibem baixa recarga e voltagem insatisfatória devido à mudança na cristalinidade (transições de fase irreversíveis) durante os ciclos de carga/descarga ${ }^{80,81}$. Atualmente, os géis de óxi- dos de vanádio porosos como $\mathrm{V}_{2} \mathrm{O}_{5}$, tanto "xerogel" como "aerogel", apresentam um alto grau de inserção de $\mathrm{Li}^{+}$, potencial e características de carga/descarga muito superiores às observadas em óxidos de metais cristalinos de composição similar ${ }^{82-84}$. $\mathrm{O} \mathrm{V}_{2} \mathrm{O}_{5}$ "xerogel” é obtido através de simples secagem evaporativa, removendo-se a água fracamente ligada à estrutura. Já o $\mathrm{V}_{2} \mathrm{O}_{5}$ "aerogel” é obtido através de secagem supercrítica; o líquido é removido do gel acima do ponto crítico onde não existe tensão superficial. A vantagem da estrutura "aerogel" reside no fato de que a escala de porosidade pode ser controlada e manipulada, e a rede sólida se mantém interconectada.

Entre os materiais catódicos utilizando o óxido de vanádio, um dos mais promissores para dispositivos secundários de lítio foi reportado recentemente por Gil et al. ${ }^{85}$. Nesse trabalho, $\mathrm{o} \mathrm{Li}_{4} \mathrm{~V}_{3} \mathrm{O}_{7,9}$ foi preparado a partir da redução do vanádio com sulfito e os resultados apontaram uma capacidade de $220 \mathrm{~A} \mathrm{~h} \mathrm{~kg}^{-1}$, além de uma longa vida (superior a 100 ciclos de carga/descarga) e energia específica de $630 \mathrm{Wh} /$ $\mathrm{kg}$, superior a outros materiais com o $\mathrm{Li}_{3} \mathrm{NaV}_{3} \mathrm{O}_{8}^{86}$, por exemplo.

Considerando-se as rotas alternativas à preparação dos materiais tidos como "convencionais", Patrissi et al. ${ }^{87}$ sintetizaram nanofios de $\mathrm{V}_{2} \mathrm{O}_{5}$ pela deposição de um precursor alcóxido nos microporos de uma membrana "template" de policarbonato. Neste trabalho, os autores estudaram as capacidades de carga de eletrodos nanoestruturados e compararam os valores com os obtidos com eletrodos de filmes finos, contendo o mesmo tipo e quantidade de material eletródico. Experimentos de descarga galvanostática mostraram que a maiores correntes de descarga $\left(13,3 \mathrm{~mA} \mathrm{~cm}^{-2}\right.$ para eletrodos nanoestruturados e $11,4 \mathrm{~mA} \mathrm{~cm}^{-2}$ para eletrodos de filmes finos), os eletrodos nanoestruturados apresentam capacidades maiores que eletrodos de filmes finos. Isto ocorre porque nos eletrodos nanoestruturados, as distâncias do caminho difusional dos íons $\mathrm{Li}^{+}$são mais curtas e a área superficial é maior. Já para menores correntes de descarga, a capacidade de carga/descarga é equivalente para ambos os eletrodos. Ainda nessa linha, outro trabalho reportado recentemente trata da obtenção de nanotubos de $\mathrm{V}_{2} \mathrm{O}_{5}$ por Novák et al.$^{88}$, através da síntese de sol-gel utilizando moléculas de surfactantes como "template". Apesar da carga específica diminuir com o aumento do número de ciclos (180 $\mathrm{mA} \mathrm{h} \mathrm{g}^{-1}$ no primeiro ciclo e $100 \mathrm{~mA} \mathrm{~h} \mathrm{~g}^{-1}$ no oitavo ciclo), o desenvolvimento desse novo material nanoestruturado apresenta-se como um desafio bastante atraente, visto que a forma tubular favorece a uma cinética mais rápida de inserção/deinserção de $\mathrm{Li}^{+}$.

\section{Materiais Orgânicos: Polímeros Condutores Eletrônicos (PCEs) e Eletrodos Sólidos de Polimerização Redox (ESPR) ou Organo Tióis}

A partir de 1979, quando foi mostrado que os processos eletroquímicos direto e inverso de dopagem podiam ser obtidos de maneira reversível ${ }^{89}$, muitas pesquisas têm se desenvolvido em novos tipos de baterias secundárias utilizando polímeros condutores eletrônicos (PCEs) como materiais eletroativos ${ }^{90-96}$. Em linhas gerais, pode-se citar as características abaixo como particularmente necessárias a um polímero candidato a ser utilizado como eletrodo em dispositivos secundários ${ }^{97}$,

(a) Alta quantidade de dopante por unidade de massa e unidade de volume;

(b) Boa reversibilidade nos processos direto e inverso de dopagem;

(c) Difusão rápida de dopante na matriz polimérica;

(d) Alto potencial para dopante do tipo- $p$ e baixo para dopante do tipo- $n$;

(e) Estabilidade química, eletroquímica e térmica e facilidade de manuseio;

(f) Estabilidade em relação à solução do eletrólito;

(g) Alta condutividade. 
A bateria deve satisfazer tantos requisitos quantos forem possíveis dos citados acima, no sentido de obter boa capacidade, eficiência e ciclabilidade, além de características de armazenamento.

Das seis configurações possíveis para baterias utilizando polímeros condutores $^{98}$, três delas utilizam o cátion produzido pela descarga do ânodo para a compensação de cargas no cátodo. Tais configurações apresentam, entre outras, a vantagem de não necessitar de grandes quantidades de eletrólito, utilizando freqüentemente membranas finas com polímeros condutores iônicos, o que diminui a massa e o volume da bateria, aumentando sua capacidade específica.

Entre os polímeros condutores eletrônicos, a polianilina, PANI, se destaca devido às suas propriedades como estabilidade química em condições ambientais, processabilidade, facilidade de polimerização e dopagem, baixo custo, entre outras ${ }^{99}$. Considerando a contribuição iônica ao processo de compensação de cargas, sabe-se que na polianilina, a compensação de cargas é feita principalmente pelos ânions ${ }^{100}$. Este processo está associado a uma degradação na densidade de energia, resultando em capacidade inferior a $30 \mathrm{~W} \mathrm{~h} \mathrm{~kg}^{-1}$, para o sistema PANI-Li ${ }^{101}$. Outro fato importante é a mudança estrutural que ocorre no material durante o processo redox, como o inchamento devido à entrada de solvente.

Têm sido obtidos vários derivados da polianilina, manufaturados através da substituição no monômero original, produzindo novos materiais ativos com propriedades físicas distintas da polianilina $^{102-105}$. Um dos tipos mais interessantes de derivados são as chamadas polianilinas auto-dopadas; tais polímeros contêm grupos funcionais aniogênicos, aptos a interagir com átomos de nitrogênio positivamente carregados presentes na cadeia de polianilina, causando novos efeitos, diferentes daqueles apresentados por polímeros dopados com ânions em solução ${ }^{106}$. Portanto, a transformação de um polímero que troca fundamentalmente ânions em um que troque cátions pode ser alcançada através da incorporação irreversível de grupos carregados negativamente na cadeia polimérica. É importante que a massa adicionada seja mínima (grupos aniônicos leves), no sentido de manter a carga específica com um valor relativamente alto.

Na Figura 2 estão mostradas duas configurações: na situação (a) o polímero empregado no cátodo utiliza predominantemente ânions no processo de compensação de cargas e necessita de um reservatório para armazenar os cátions liberados pelo ânodo e os ânions expelidos pelo cátodo; para a situação (b), a compensação de cargas é feita por cátions liberados pelo ânodo e o eletrólito pode ser uma membrana fina, em lugar do reservatório requerido em (a). Além das configurações para cada situação, são mostrados dois esquemas reacionais para a polianilina e uma derivada autodopada, denotada genericamente pela presença do grupo $-\mathrm{SO}_{3}{ }^{-}$como no caso das conhecidas SPAN ${ }^{94,107,108}$ (polianilina sulfonada) e PAPSAH ${ }^{109-111}$ (polianilina-co-N-ácido propanossulfonico). Através das configurações ilustradas, é fácil notar que a utilização de um material que troque fundamentalmente cátions com a fase eletrolítica apresenta vantagem adicional, pois resulta em baterias de tamanho menor com consequente incremento de todas as suas propriedades específicas ${ }^{90}$.

A respeito das reações mostradas na Figura 2, é importante enfatizar que se tratam de esquemas ideais, já que não consideram as participações de cátions e ânions além de eventuais moléculas de solvente. A investigação da natureza dessas espécies é de fundamental importância na avaliação real das propriedades específicas do material. Nesse sentido, a microbalança eletroquímica a cristal de quartzo (MECQ) vem sendo utilizada com relativo sucesso na descrição das espécies iônicas que cruzam a interface filme/solução ${ }^{112}$. As Figuras 3 e 4 comparam as reações redox para a PANI ${ }^{113}$ e sua derivada autodopada com o grupo sulfônico PAPSAH ${ }^{114}$, respectivamente, sob condições potenciodinâmicas a $50 \mathrm{mVs}^{-1}$ em soluções de a)

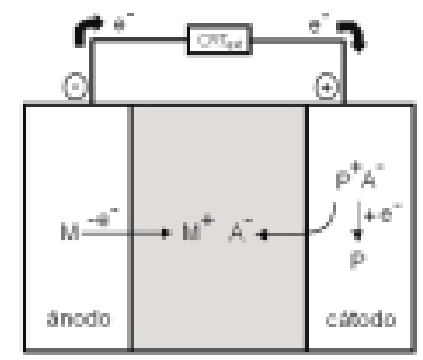

$\mathrm{PANI}+\alpha \mathrm{ClO}_{4}^{-}+\alpha \mathrm{Li}^{+} \Leftrightarrow \mathrm{PANI}^{\alpha+}, \alpha \mathrm{ClO}_{4}^{-}+\alpha \mathrm{Li}$

b)

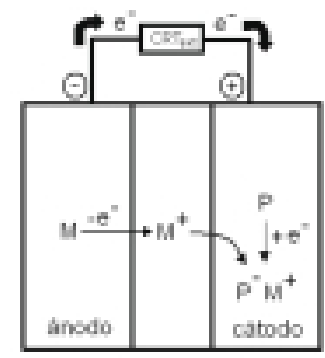

$\mathrm{PANI}-\alpha \mathrm{SO}_{3}^{-}, \alpha \mathrm{Li}^{+} \Leftrightarrow \mathrm{PANI}^{\alpha+}-\alpha \mathrm{SO}_{3}^{-}+\alpha \mathrm{Li}^{+}$

$(\rightarrow)$ Carga e $(\leftarrow)$ descarga

Figura 2. Descarregamento e compensação de cargas em baterias recarregáveis com cátodo polimérico. a) Compensação de cargas feita majoritariamente por ânions e b) compensação feita majoritariamente por cátions. Adaptado de ref. [98]

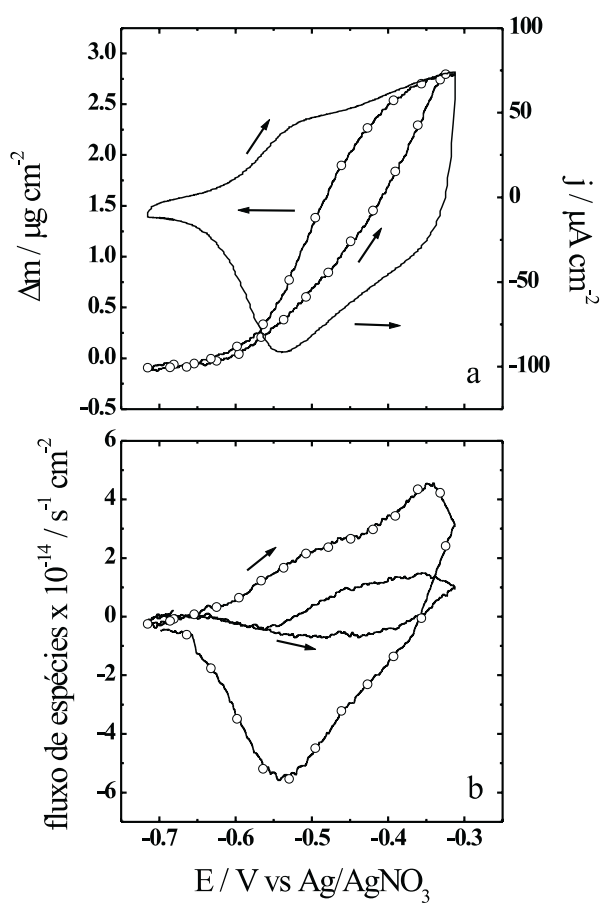

Figura 3. a) Perfis de corrente (-) e variação de massa (-O-O-) em função do potencial e b) fluxo molar de cátions (-) e ânions (-O-O-) em função do potencial para um eletrodo modificado com PANI em solução de $\mathrm{LiClO}_{4} / \mathrm{ACN}$. $v=50 \mathrm{mVs}^{-1}$. Adaptado de ref. [113] 

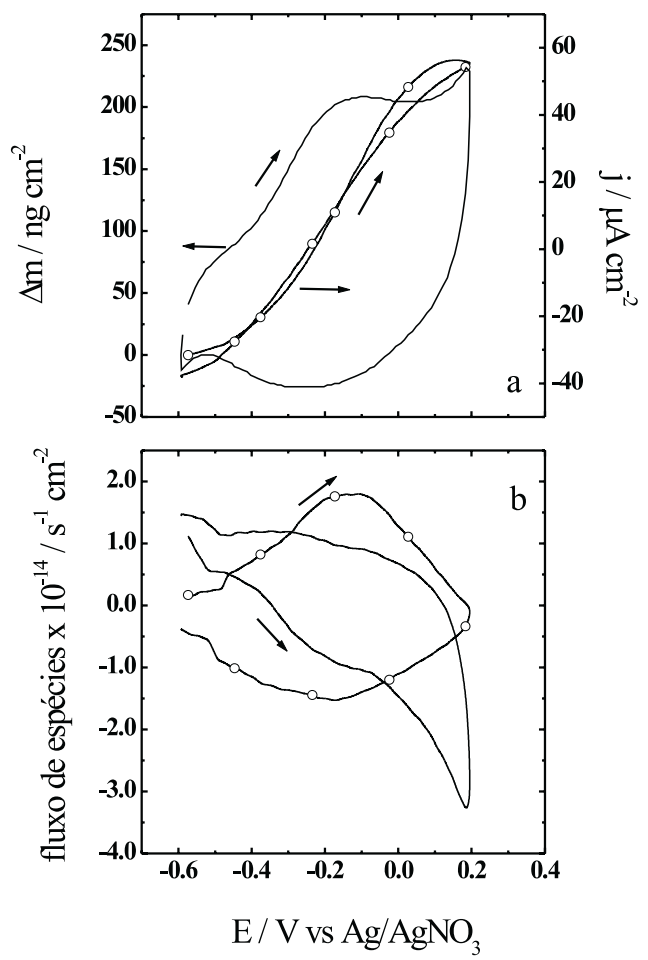

Figura 4. a) Perfis de corrente (-) e variação de massa (-O-O-) em função do potencial e b) fluxo molar de cátions (-) e ânions (-O-O-) em função do potencial para um eletrodo modificado com PAPSAH em solução de $\mathrm{LiClO}_{4} / \mathrm{ACN}$. $v=50 \mathrm{mVs}^{-1}$. Adaptado de ref. [114]

$\mathrm{LiClO}_{4}$ /acetonitrila (ACN). Em ambos os casos são mostrados os perfis de corrente, $\mathrm{j}$, e variação de massa, $\Delta \mathrm{m}$, além das curvas com os fluxos iônicos de $\mathrm{Li}^{+}$e $\mathrm{ClO}_{4}^{-}$somados às contribuições de solvente. Nas duas situações, o perfil de massa é dominado pela inserção de ânions em base mássica durante a varredura positiva. No entanto, analisando-se os fluxos das espécies, observa-se que no eletrodo modificado com PANI, Figura 3, a compensação de cargas é atingida com participação massiva de ânions, além do solvente também envolvido. Em contraste, a inclusão do grupo sulfônico na cadeia original altera as características da PANI e a troca de cátions é responsável por aproximadamente $50 \%$ do processo de eletroneutralização da PAPSAH, Figura 4, sem mencionar a menor participação de moléculas neutras (solvente) no caso do eletrodo modificado com o polímero auto-dopado. A participação de cátions no caso da PAPSAH foi discutida em termos da diminuição de mobilidade experimentada pelos íons $\mathrm{Li}^{+}$em virtude da formação de pares iônicos com os sítios aniônicos fixos na cadeia da PAPSAH ${ }^{106}$. Em resumo, observou-se que as reações descritas anteriormente, Figura 2, não se aplicam ao caso da PAPSAH e a participação dual de cátions e ânions durante o processo de compensação de cargas deve ser levada em conta, assim como as condições de eletroneutralidade da fase eletrolítica visando a configuração do dispositivo como discutido anteriormente.

Um fato adicional a ser considerado na inclusão de grupos à cadeia da PANI é o aumento de solubilidade que melhora a processabilidade do polímero original. Ao lado das tradicionais polianilinas sulfonadas como SPAN e PAPSAH outras, utilizando grupos como metóxi e etóxi ${ }^{115-117}$, ácido benzoilsulfônico $(\mathrm{PABSAH})^{118}$, ácido propil-benzenossulfônico (PAPBSAH) $)^{119}$, têm sido estudadas. No entanto, deve estar claro o compromisso entre as propriedades a serem modificadas ${ }^{120,121}$. Como exemplo, Ito et al. ${ }^{122}$ mostraram que o aumento do grau de sulfonação da PANI aumenta a solubilidade, mas é sempre acompanhado por uma diminuição da condutividade.

Ao lado das vantagens aliadas à utilização de PCEs em dispositivos secundários alguns problemas básicos também devem ser considerados:

(a) Lenta taxa de carga-descarga: Problemas cinéticos, como difusão lenta de dopante na matriz polimérica ${ }^{123}$, conferem a tais sistemas correntes de saída da ordem de poucos $\mathrm{mA} \mathrm{cm}^{-2}$;

(b) Auto-descarga: A maioria dos polímeros utilizados apresenta uma baixa retenção de carga em eletrólitos orgânicos ${ }^{124}$. A natureza desse processo ainda não está claramente entendida, porém, a escolha adequada do solvente é crucial na estabilidade eletroquímica dos eletrodos poliméricos ${ }^{125}$;

(c) Baixas densidades de energia $\left(W h \mathrm{~kg}^{-1}\right)$ : A restrição imposta pelos baixos níveis de dopagem permitidos é uma característica marcante dos polímeros condutores eletrônicos, como o exemplo do polipirrol que pode atingir níveis de dopagem da ordem de apenas $33 \%$, ou 1 elétron por cada três repetições monoméricas $^{126-128}$. Tal limitação inerente aliada ao fato de que a operação da bateria utiliza um excesso de eletrólito (assegurando uma baixa resistência interna) conduz aos modestos valores de densidade de energia observados;

(d) Participação excessiva de solvente: A participação de solvente (ou co-intercalação) tem sido apontada como um problema capital na utilização de PCEs em dispositivos práticos ${ }^{8,90,105,106}$.

Considerando os itens (b) e (d), foi investigada a influência do solvente nas propriedades eletroquímicas de eletrodos modificados de PPY (polipirrol); particularmente estudou-se as variações de massa e carga durante condições voltamétricas ${ }^{129}$. A análise foi feita com base em uma equação do tipo Kamlet-Taft ${ }^{130}$ como reportado previamente por Otero et al. ${ }^{131}$.

A Figura 5 ilustra os resultados da carga voltamétrica e do ganho de massa no fim da varredura anódica em função dos solventes utilizados. Como se pode verificar, não há relação direta entre carga e aumento de massa, indicando que novos parâmetros devem ser incluídos na análise dos fatores que influenciam ambas as propriedades. Assim, os resultados de carga foram racionalizados em termos de algumas propriedades dos solventes utilizados, como acidez e basicidade, polarizabilidade e constante dielétrica ${ }^{132}$. Já para a análise das variações de massa foram utilizados todos os parâmetros mencionados na análise da carga além de propriedades de transporte (especificamente número de transporte aparente de $\mathrm{Li}^{+}$) e volume das moléculas do solvente. As correlações obtidas justificaram a introdução dos parâmetros estérico e de transporte no modelo e ambas as

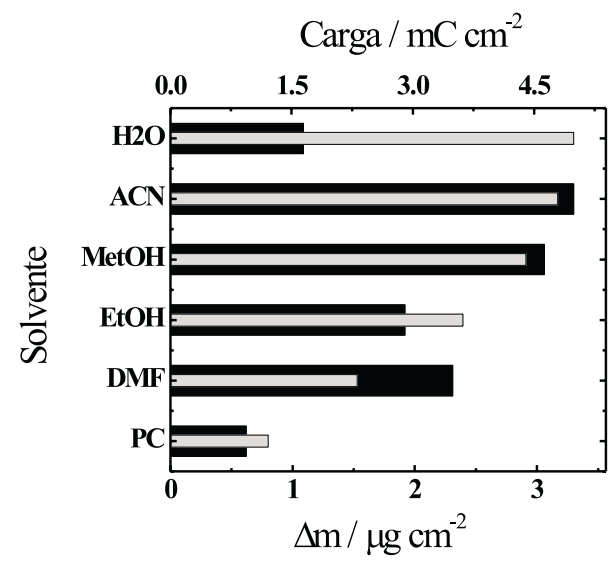

Figura 5. Gráficos em barras para ganho de massa e carga em função do solvente para eletrodos modificados de polipirrol. Adaptado de Ref. [129] 
propriedades (carga armazenada e aumento de massa) foram relacionadas às predições teóricas através de multiregressão com um ajuste superior 0,994 em todos os casos.

Em geral, a faixa de aplicação dos dispositivos contendo eletrodos poliméricos é apreciavelmente limitada. De fato, esses dispositivos encontram maiores aplicações em sistemas pequenos em microeletrônica. No entanto, a utilização de dispositivos com esses materiais representa um importante avanço, não somente sob o ponto de vista tecnológico, mas particularmente devido à redução da poluição ambiental por metais pesados provenientes da utilização das células secas mais comuns. Compósitos entre PCEs são basicamente formados com objetivo de se obter um material do tipo-n em lugar de polímeros tipo-p (como PANI e PPY, por exemplo), incrementando a participação de cátions na compensação de cargas, diminuindo o tamanho do dispositivo e aumentando o valor das suas propriedades específicas, como discutido para os polímeros autodopados. O poli(estireno sulfonato) tem sido utilizado como compósito tanto com PANI ${ }^{133}$ quanto com PPY ${ }^{134}$. Momma et al. ${ }^{86}$, através de medidas de impedância, observaram que maiores coeficientes de difusão para o $\mathrm{ClO}_{4}^{-}$são obtidos no compósito PPY/Nafion, em comparação com o PPY. As medidas foram realizadas em estado sólido, utilizando-se o sistema Li/PEO (poli(óxido de etileno))$\mathrm{LiClO}_{4} / \mathrm{PPY}$ e Li/PEO-Li ClO $/$ (PPY/Nafion).

Outro caso interessante a ser discutido é o aumento de porosidade com a utilização de polímero depositado sobre uma matriz fibrosa de grafite, que apresenta uma estrutura leve e alta área superficial. Coffey et al. ${ }^{135}$ obtiveram resultados satisfatórios depositando eletroquimicamente o PPY sobre uma matriz de grafite, com um aumento considerável na densidade de carga do sistema. Tecido de carbono (PWB-3, Stackpole/E-tek) foi recentemente utilizado como substrato para o cátodo polimérico de PANI ${ }^{136}$. Tal material tem sido empregado com sucesso em eletrodos de difusão de gás para células a combustível com eletrólito polimérico sólido ${ }^{137-139}$. Sua utilização como substrato em cátodos poliméricos para dispositivos secundários representa uma alternativa bastante atraente quando considerados fatores como baixo custo, facilidade de manuseio e flexibilidade do cátodo formado. A Figura 6 ilustra a micrografia do tecido de carbono modificado com $0,43 \mathrm{mg}$ PANI eletroquimicamente formada, através da qual fica explícito o aumento de área superficial quando comparada com outros eletrodos.

A avaliação das propriedades do cátodo PANI/tecido de carbono foi feita utilizando $\mathrm{LiClO}_{4} /$ carbonato de propileno (PC) e lítio metálico com ânodo. Utilizando correntes de $0,1 \mathrm{~mA}$ e $-0,1 \mathrm{~mA}$, foram

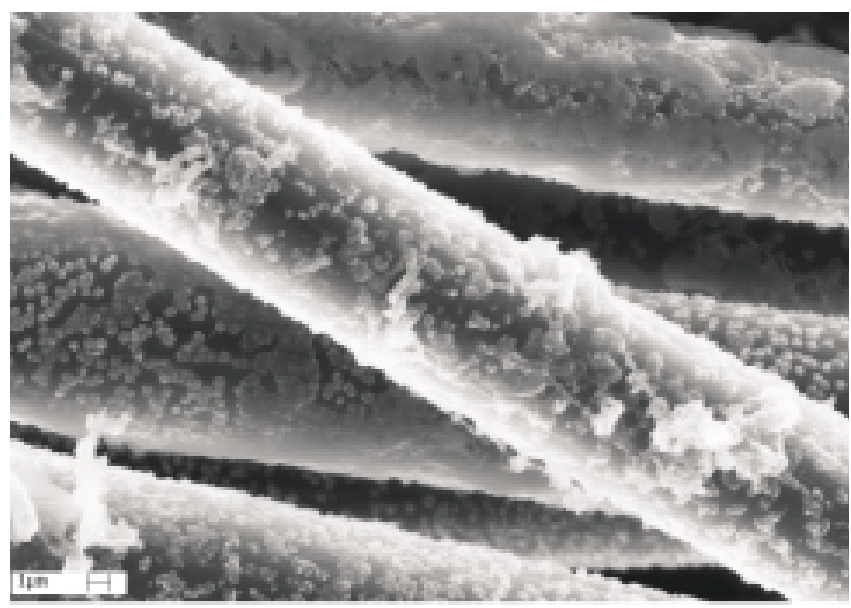

Figura 6. Micrografias do tecido de carbono após deposição eletroquímica da PANI com um aumento de 10.000 vezes realizados experimentos de carga e descarga entre 2 e $4 \mathrm{~V}$ versus $\mathrm{Li}^{+} /$ Li. Em todos os ciclos, a eficiência coulômbica foi muito próxima a $100 \%$. A Figura 7 ilustra a capacidade em função do número de ciclos de carga e descarga, a capacidade atinge um valor constante em torno do décimo ciclo, chegando a $70 \mathrm{~A} \mathrm{~h} \mathrm{~kg}^{-1}$ no último, o que representa cerca de $88 \%$ do valor observado no primeiro processo de intercalação e deintercalação de $\mathrm{Li}^{+}$. Os resultados obtidos nesse trabalho são superiores ao obtido para o sistema em estado sólido utilizando $\mathrm{LiClO}_{4}$ - polióxido de etileno como eletrólito (PANI/ $\left.\mathrm{LiClO}_{4}-\mathrm{POE} / \mathrm{Li}\right)^{140}$, e ao reportado por Matsunaga et al. ${ }^{141}$, sendo porém, inferiores ao reportado por Morita et al. ${ }^{142}\left(\mathrm{PANI}_{\mathrm{LiClO}}{ }_{4}^{-}\right.$ PC+DME (1,2-dimetoxi-etano)/Li). Estes últimos autores utilizaram a PANI depositada sobre eletrodo de platina.

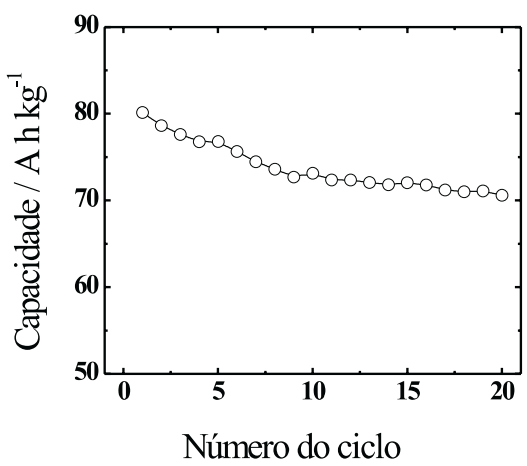

Figura 7. Capacidade em função do número de ciclos para o protótipo contendo PANI

A classe de materiais catódicos denominada eletrodos sólidos de polimerização redox (ESPR) que fazem uso de organo tióis tem atraído considerável atenção recentemente. Os organo tióis e dissulfitos constituem uma classe de materiais com um importante papel em bioquímica, tendo em vista que a configuração de proteínas como a ribonucleonase é mantida através da ligação dissulfito ${ }^{143,144}$. A Figura 8 ilustra alguns destes compostos estudados por Liu et al. ${ }^{145}$.

Os processos de formação e quebra da ligação do dissulfito estão relacionados ao carregamento e descarregamento em sistemas de
$\left\{\mathrm{SCH}_{2} \mathrm{CH}_{2} \mathrm{OCH}_{2} \mathrm{CH}_{2} \mathrm{~S}\right\}_{\mathrm{n}}$

2 - mercaptoetil éter<smiles>CC(C)(C)[SiH2]CCSC(C)(C)C</smiles>

1,2 - etanodiol<smiles>C1CSC2(C1)CCN(CCN1SCCS1)S2</smiles>

etilenodiamina

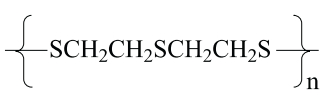

2 - mercaptoetil sulfeto

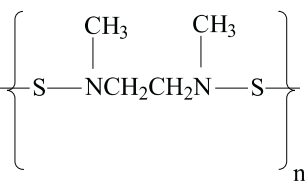

dimetil etilenodiamina
Figura 8. Exemplos de organo tióis e dissulfito utilizados como ESPR 
armazenamento ${ }^{146,147}$. Este processo pode ser exemplificado pelo par redox formado pelo 2,5 - dimercapto 1,3,4 - tiadiazol (DMcT) e seu polímero poli-[2,5 - dimercapto (1,3,4 - tiadiazol)] (PDTT), ilustrado na Figura 9.<smiles>Sc1nnc(S)s1</smiles>
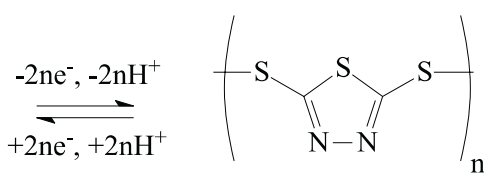

Figura 9. Par redox formado pelo DMcT e PPDT

Assim, os processos de carregamento e descarregamento de energia resultam da reação de transferência eletrônica entre a formação e quebra da ligação do dissulfito. Quando tióis bifuncionais com o DMcT são utilizados como material catódico, o polímero é formado durante o carregamento e o descarregamento regenera a forma monomérica inicial. Ambos os processos direto e inverso de polimerização foram recentemente estudados através do emprego da microbalança eletroquímica a cristal de quartzo e espectroeletroquímica ${ }^{148}$. Como vantagens dos organo tióis pode-se destacar o baixo custo e a desprezível toxicidade, como o DMcT.

Entre os compósitos utilizando polímeros condutores, pode-se afirmar que um dos mais bem sucedidos é o formado entre a PANI e o 2,5-dimercapto-1,3,4-tiadiazol, DMcT, reportado inicialmente por Oyama et al. ${ }^{149}$. O emprego destes dois materiais em cátodos de baterias secundárias de lítio está baseado no efeito sinergético resultante. A primeira utilização do DMcT como material catódico foi reportada por Visco et al. ${ }^{150}$. No entanto, a utilização do DMcT puro é inviabilizada devido à cinética lenta sobre muitos materiais eletródicos a temperatura ambiente. Quando incorporado à matriz de PANI, a cinética de polimerização do DMcT é acelerada, tornando viável a utilização do material como compósito com a PANI. Adicionalmente, a PANI age como um coletor molecular de corrente para o DMcT, e este atua na prevenção da inativação do polímero acima de $4,75 \mathrm{~V}\left(v s \text {. } \mathrm{Li} / \mathrm{Li}^{+}\right)^{151}$. A Figura 10 ilustra o mecanismo envolvido durante os processos de carga e descarga do compósito PANI/DMcT, onde se observa a quebra da ligação do dissulfito concomitantemente à oxidação da PANI, compensando, dessa forma, a carga positiva recém formada na cadeia polimérica.

A capacidade experimental obtida para esse compósito foi de $185 \mathrm{~A} \mathrm{~h} \mathrm{~kg}^{-1}$, correspondendo a mais de $80 \%$ da sua capacidade total teórica (224 $\left.\mathrm{A} \mathrm{h} \mathrm{kg}^{-1}\right)$, o que indica que ambos os materiais, PANI e DMcT, são ativos durante o processo. A densidade de energia gravimétrica obtida foi em torno de $600 \mathrm{~W} \mathrm{~h} \mathrm{~kg}^{-1}$ do cátodo. Esse valor é bastante significativo se comparado com o dispositivo utili-

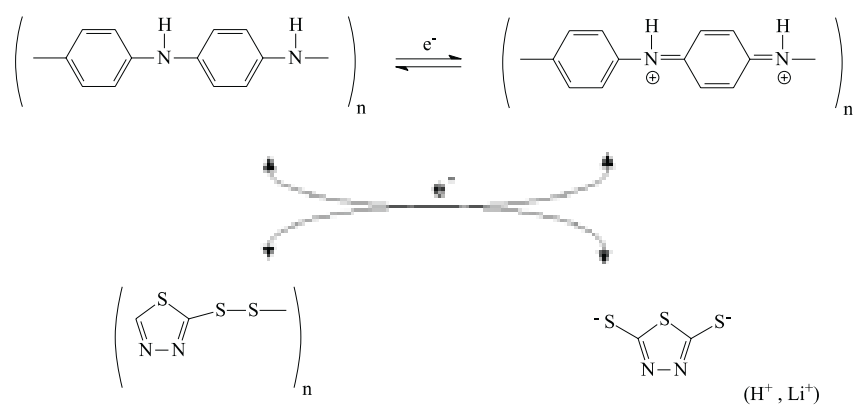

Figura 10. Mecanismo envolvido durante os processos de carga e descarga do compósito PANI/DMcT zando $\mathrm{LiCoO}_{2}$ disponível comercialmente (National/Panasonic) com aproximadamente $400 \mathrm{~W} \mathrm{~h} \mathrm{~g}^{-1} 75$.

Devido ao crescente interesse em compostos contendo DMcT, vários estudos fundamentais vêm sendo desenvolvidos no intuito de investigar os detalhes da interação entre o DMcT e a cadeia polimérica $^{152-157}$.

\section{Compósitos entre materiais orgânicos e inorgânicos}

Atualmente, muitos compósitos têm sido desenvolvidos à base de PCEs, geralmente utilizados em conjunto com outros PCEs, organo tióis e óxido metálicos, por exemplo ${ }^{158-161}$. Segundo Guinier ${ }^{162}$ : “... Supondo as propriedades (mecânicas, elétricas, etc) das diversas fases perfeitamente conhecidas, as propriedades do material compósito não são simplesmente valores médios das correspondentes aos constituintes, levando em conta as proporções respectivas. $\mathrm{O}$ material tem individualidade própria, que decorre das interações entre as fases vizinhas e das propriedades específicas das regiões de transição entre as fases ou interfaces". Dessa forma, materiais compósitos utilizando PCEs são bastante promissores no que se refere às aplicações em cátodos para dispositivos secundários de lítio.

Avanços na química de compostos de inserção foram bastante significativos no desenvolvimento de baterias recarregáveis de lítio, propiciando a produção de materiais catódicos de alta capacidade específica e densidade de energia. Entre os materiais mais estudados, podese destacar: $\mathrm{V}_{2} \mathrm{O}_{5}{ }^{163-167}, \mathrm{MnO}_{2}{ }^{168,169}, \mathrm{MoS}_{2}{ }^{170}, \mathrm{TiS}_{2}{ }^{171}$ e $\mathrm{CoO}_{2}{ }^{172}$, por exemplo.

Essas matrizes inorgânicas apresentam alguns aspectos que diminuem o desempenho da bateria. Um dos problemas encontrados nesses compostos inorgânicos é a diminuição na capacidade de carga quando o eletrodo é submetido a vários ciclos de carga e descarga, graças às variações dimensionais na matriz hospedeira devido à inserção/deinserção de solvente e íons (como por exemplo, o próprio $\mathrm{Li}^{+}$) provocando mudanças irreversíveis na estrutura. A resistência eletrônica desses materiais é relativamente baixa, proporcionando uma elevada queda ôhmica, além da lenta difusão dos íons $\mathrm{Li}^{+}$ dentro da estrutura, responsável pela diminuição da capacidade específica e densidade de energia da bateria ${ }^{62,173}$.

Essas matrizes hospedeiras uni e bidimensionais, constituídas de camadas, canais, túneis ou completamente amorfas, representam uma classe de material, cuja estrutura pode se adaptar às dimensões de outras espécies intercalantes. Sistemas híbridos constituídos de macromoléculas orgânicas e matrizes inorgânicas apresentam propriedades peculiares em razão da íntima mistura em escala nanométrica entre os seus componentes, correspondendo a uma escala intermediária entre a molecular clássica e microscópica. Assim, mudanças volumétricas na estrutura do cátodo durante o processo redox podem ser evitadas com a inserção de determinados polímeros na região interlamelar. Pode-se obter, também, um aumento na condutividade elétrica ao se intercalar polímeros condutores eletrônicos, principalmente em sistemas que possibilitam o aumento na linearidade das cadeias ${ }^{174}$. Existem vários métodos de obtenção dessas nanoestruturas, sendo alguns deles: a polimerização de moléculas do monômero na rede inorgânica através de um tratamento químico, térmico ou fotoinduzido in situ; a intercalação do polímero previamente formado e através de uma polimerização intercalativa redox durante a preparação do óxido pelo método sol-gel, onde os monômeros são oxidados pelo material inorgânico, o qual se reduz e promove a formação do polímero entre as suas camadas ${ }^{175}$. PANI ${ }^{176}$, $\mathrm{PPy}^{177}$, politiofeno ${ }^{178}$ (PTH), e poli(óxido de etileno) ${ }^{179}$ compreendem alguns dos polímeros inseridos em matrizes hospedeiras.

Em 1987, foi obtido o primeiro nanocompósito envolvendo PCE em um material bidimensional, onde o PPy foi inserido nas lamelas 
do $\mathrm{FeOCl}$ via redução do $\mathrm{Fe}^{3+}$. A formação dessa mistura com um íntimo contato entre os seus componentes foi verificada pelo au-

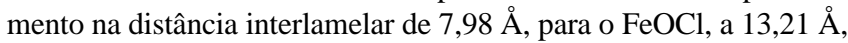
quando da formação do nanocompósito com o PPy ${ }^{180}$. A inserção de PANI também foi observada a partir dos dados de difração de raio-X, proporcionando um aumento de 5,94 $\AA$ entre as camadas de $\mathrm{FeOCl}$, suficiente para acomodar uma camada do polímero ${ }^{181,182}$. Esses materiais apresentaram uma condutividade elétrica superior a outros compostos de intercalação envolvendo o composto inorgânico, em razão da presença do polímero condutor eletrônico.

Outro aspecto importante desses nanocompósitos está relacionado à ciclabilidade desses materiais, quando utilizados como cátodos em baterias secundárias. Por exemplo, quando um cátodo de $\mathrm{FeOCl}$ é submetido a um processo catódico na presença de carbonato de propileno e $\mathrm{LiBF}_{4}$, o produto de descarga se decompõe, diminuindo a ciclabilidade da bateria. No entanto, a formação de novos materiais provenientes da reação entre os monômeros de anilina e $\mathrm{FeOCl}$ na presença de $\mathrm{H}_{2} \mathrm{O}$, possibilitaram uma maior recarregabilidade do cátodo devido à inserção/deinserção reversível de íons $\mathrm{Li}^{+}$na sua estrutura $^{183}$.

O compósito constituído de $\mathrm{MnO}_{2}$ e PPy foi preparado no sentido de aumentar a baixa condutividade elétrica do óxido, substituindo o pó de carbono normalmente utilizado para esse fim. Uma vez que o polímero é eletroquimicamente ativo, o compósito constituído de $15 \%$ de PPy sobre a massa total do cátodo apresentou propriedades eletroquímicas superiores em relação ao filme dióxido de manganês contendo carbono negro na mesma estequiometria. Contudo, apesar do processo redox sob o PPy/MnO 2 envolver a participação de $\mathrm{Li}^{+}$na compensação de cargas do óxido, verificou-se a participação dos íons $\mathrm{ClO}_{4}^{-}$nas etapas de oxidação/redução do material orgânico ${ }^{184,185}$, como mostra a Figura 11. Acredita-se que o aumento na quantidade de ânions no filme de $\mathrm{PPy} / \mathrm{MnO}_{2}$ em relação ao PPy se deve aos íons $\mathrm{Cl}^{-}$adsorvidos sobre as partículas de $\mathrm{MnO}_{2}$ durante a preparação do material. Esse mecanismo proposto implica em uma alteração na concentração do eletrólito, desfavorecendo a utilização do compósito em baterias de alta densidade energética, como discutido anteriormente ${ }^{90}$.

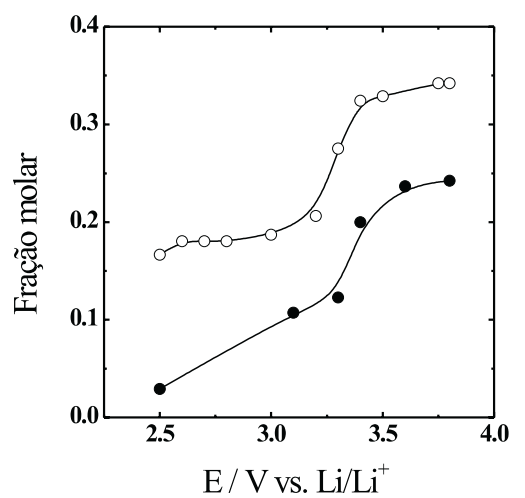

Figura 11. Fração molar de ânions $\left(\mathrm{Cl}^{-}\right.$e $\left.\mathrm{ClO}_{4}^{-}\right)$incorporados no filme de (O) PPy e (•) $\mathrm{MnO}_{2}$ / PPy para diferentes potenciais em uma solução de 1,0 $\mathrm{M} \mathrm{LiClO}_{4}$ em carbonato de propileno/1,2 dimetoxietano (1:1). Adaptada da ref. [184]

A PANI foi incorporada a materiais como $\mathrm{HMWO}_{6}$ (nos quais, $\mathrm{M}=\mathrm{Ta}, \mathrm{Nb}$ ), cuja acidez de Brönsted promoveu a formação de nanocompósitos. No entanto, o potencial redox dessas matrizes não favorece a oxidação dos monômeros intercalados, necessária para a sua polimerização. Aquecendo o material híbrido a $130{ }^{\circ} \mathrm{C}$ por $48 \mathrm{~h}$ na presença do ar atmosférico, ocorreu a transformação de [anilina $]_{0,68} \mathrm{HMWO}_{6}$ a $[\mathrm{PANI}]_{0,34} \mathrm{HMWO}_{6}$, na qual apenas metade dos monômeros se polimerizaram, sendo a outra metade expulsa. Ao determinar os valores dos coeficientes de difusão dos íons $\mathrm{Li}^{+}$durante o processo de carga/descarga, verificou-se que a mobilidade do íon $\mathrm{Li}^{+}$aumentou significativamente na estrutura do nanocompósito em relação à matriz puramente inorgânica. Esse fato foi atribuído a uma diminuição na interação entre o íon intercalante e a rede hospedeira devido à presença da PANI, otimizando o processo de transferência de massa ${ }^{186}$.

Em razão da alta capacidade específica do xerogel $\mathrm{V}_{2} \mathrm{O}_{5}$, vários nanocompósitos desse óxido foram sintetizados e caracteriza$\operatorname{dos}^{171,187-189}$. Um exemplo foi a formação do híbrido entre o tetratiofulvaleno (TTF) e o pentóxido de vanádio, cuja inclusão foi obtida através de um mecanismo de transferência de carga, também verificado para o sistema constituído à base de benzidina $(\mathrm{Bz})^{190}$. Moléculas de piridina $(\mathrm{Pi})$ intercaladas na estrutura do óxido foram analisadas pela técnica de absorção de raio-X (EXAFS) e infravermelho, indicando a formação de uma ligação de coordenação entre Pi e o átomo de vanádio, através de uma ligação de Lewis ${ }^{191}$.

A intercalação de monômeros de polímeros condutores como anilínio $\left(\mathrm{C}_{6} \mathrm{H}_{5} \mathrm{NH}_{3}^{+}\right)$é relativamente rápida, propiciando a formação de PANI na matriz inorgânica hospedeira. O mecanismo de oxidação do monômero, necessário para o processo de polimerização, foi analisado a partir da inserção de $\mathrm{C}_{6} \mathrm{H}_{5} \mathrm{NH}_{3}{ }^{+} \mathrm{I}^{-}$no óxido que, posteriormente, foi submetido ao ar atmosférico. Como esse sal praticamente não é oxidado à polianilina quando exposto ao oxigênio atmosférico, concluiu-se que $\mathrm{V}_{2} \mathrm{O}_{5}$ atua como um catalisador durante a polimerização, a qual está intimamente associada à transferência de elétrons para o oxigênio molecular ${ }^{192}$. Quando a PANI é intercalada a partir dos monômeros de anilina, observa-se uma expansão de aproximadamente 2,39 A entre as camadas do óxido, que passam de

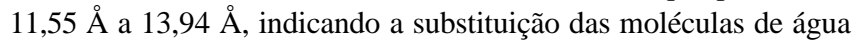
pelo polímero na região interplanar. Além disso, a condutividade elétrica desses materiais aumentou em torno de 4 ordens de magnitude em relação ao xerogel de $\mathrm{V}_{2} \mathrm{O}_{5}{ }^{184}$.

$\mathrm{O}$ primeiro estudo envolvendo a intercalação de íons $\mathrm{Li}^{+} \mathrm{em}$ nanocompósitos, constituídos à base de polímeros condutores eletrônicos e óxido de vanádio, foi realizado recentemente por Nazar et $a l .{ }^{193}$. Nesse estudo, a PANI foi sintetizada na forma esmeraldina, a qual foi formada pela polimerização oxidativa do óxido, reduzindo os centros $\mathrm{V}(\mathrm{V})$ a $\mathrm{V}(\mathrm{IV})$. Depois de um tratamento pós-oxidativo à base de $\mathrm{O}_{2}$ a $150{ }^{\circ} \mathrm{C}$ por $8 \mathrm{~h}$, visando a reoxidação dos sítios $\mathrm{V}(\mathrm{IV})$, houve um ganho de aproximadamente $40 \%$ na capacidade específica em relação ao óxido puro na presença de $\mathrm{LiClO}_{4}$ em carbonato de propileno. $\mathrm{O}$ polímero proporcionou uma difusão iônica mais rápida e uma boa reversibilidade. De acordo com Romero et al. ${ }^{194}$, $\left[\mathrm{C}_{6} \mathrm{H}_{4} \mathrm{~N}\right]_{0.6} \mathrm{~V}_{2} \mathrm{O}_{5} .0,3 \mathrm{H}_{2} \mathrm{O}$ depois do tratamento pós-oxidativo à base de $\mathrm{O}_{2}$ apresentou uma inserção de lítio aproximadamente 4,5 vezes maior de que $\mathrm{V}_{2} \mathrm{O}_{5}$ seco a $100{ }^{\circ} \mathrm{C}$.

A PANI possui a vantagem de ser menos sensível à oxidação química do que outros polímeros como o PPY, permitindo que determinados nanocompósitos sejam pós-oxidados, cujas respostas eletroquímicas são superiores à soma dos seus componentes separadamente ${ }^{195}$. Mesmo não sendo estáveis ao tratamento à base de $\mathrm{O}_{2}$, os compósitos constituídos à base de $\mathrm{PPY} / \mathrm{V}_{2} \mathrm{O}_{5}$ e $\mathrm{PTH} / \mathrm{V}_{2} \mathrm{O}_{5}$ quando submetidos inicialmente a um processo de carga apresentam também valores consideráveis da capacidade específica ${ }^{196}$. Porém, suas respostas eletroquímicas dependem bastante do método de preparação. Quando o polímero se encontra em excesso na superfície, ele bloqueia a entrada de íons $\mathrm{Li}^{+}$nas regiões interplanares, considerando a mesma estrutura e quantidade de $\mathrm{V}(\mathrm{V})$ no filme.

Quando o composto inorgânico não atua como contra-ânion para 
o material orgânico, uma alternativa para evitar a participação de ânions é a produção de materiais híbridos constituídos de polímeros condutores auto-dopados. Um compósito formado à base da polianilina sulfonada SPAN e $\mathrm{MnO}_{2}$ (ramsdellita) ainda apresentou uma capacidade de descarga maior do que cátodos de SPAN e uma melhor ciclabilidade em relação ao óxido puro ${ }^{197}$.

Outro exemplo desses sistemas é a formação de cátodos constituídos de $\mathrm{V}_{2} \mathrm{O}_{5}$ e um derivado completamente sulfonado da polianilina, poli(anilina ácido $\mathrm{N}$-propano sulfônico), denominado PSPAN $^{198}$. Os compósitos foram obtidos através do método sol-gel no qual o precursor (triisopropóxido de vanádio) foi adicionado a uma solução aquosa de anilina ácido N-propano sulfônico. Dessa forma, obteve-se o nanocompósito PSPAN $/ \mathrm{V}_{2} \mathrm{O}_{5}$ através de uma polimerização do monômero e da hidrólise e condensação do alcóxido de vanádio. Foi adicionado $\mathrm{H}_{2} \mathrm{O}_{2}$ ou borbulhado $\mathrm{O}_{2}$ na solução para maximizar a capacidade específica do material, proporcionando um aumento de $30 \%$ em relação ao $\mathrm{V}_{2} \mathrm{O}_{5}$ em carbonato de propileno contendo 0,5 $\mathrm{M}$ de $\mathrm{LiClO}_{4}$. A Figura 12 ilustra as curvas de cronopotenciometria dos filmes $\mathrm{V}_{2} \mathrm{O}_{5}$ (linha pontilhada), PSPAN/ $\mathrm{V}_{2} \mathrm{O}_{5}$ (linha tracejada) e $\mathrm{H}_{2} \mathrm{O}_{2}$-PSPAN/ $\mathrm{V}_{2} \mathrm{O}_{5}$ (linha sólida) sob uma densidade de corrente constante de $-10 \mu \mathrm{A} / \mathrm{cm}^{2}$. Apesar da menor capacidade específica em relação ao nanocompósito PANI/ $\mathrm{V}_{2} \mathrm{O}_{5}$, apenas $\mathrm{Li}^{+}$participou do processo de compensação de carga, evitan-

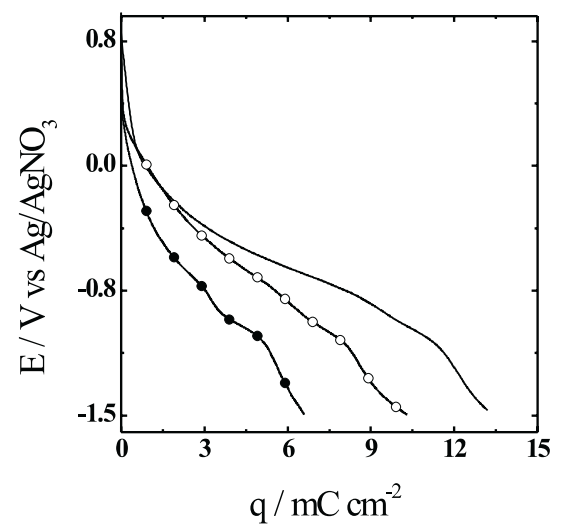

Figura 12. Variação do potencial em função da intercalação de $\mathrm{Li}^{+}$nos

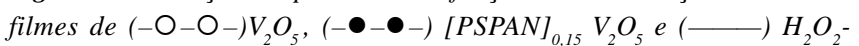
$[P S P A N]_{0,15} V_{2} \mathrm{O}_{5}$ em $0.5 \mathrm{M} \mathrm{de} \mathrm{LiClO}_{4} / \mathrm{PC} . j=10 \mathrm{~mA} / \mathrm{cm}^{2}$. Massa da amostra = 12,1 mg. Adaptada da ref.[198]

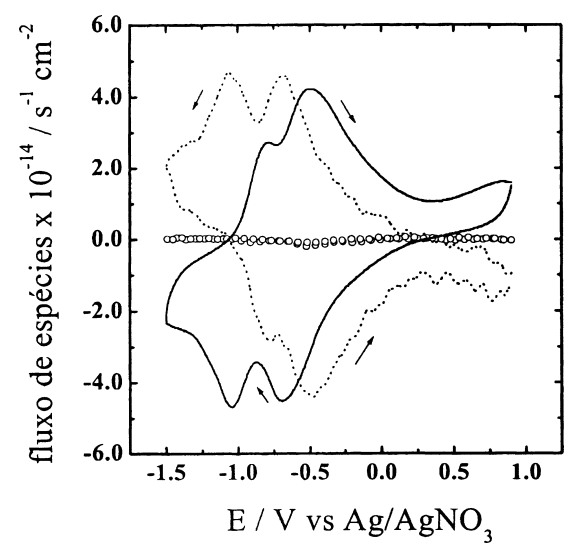

Figura 13. Fluxo de $\mathrm{Li}^{+}(-\bullet-\bullet), \mathrm{ClO}_{4}^{-}$e moléculas de PC (O) e elétrons (—) no filme de [PSPAN $]_{0,15} V_{2} \mathrm{O}_{5}$ durante o processo redox em $0.5 \mathrm{M}$ de $\mathrm{LiClO}_{4} / \mathrm{PC} . v=5 \mathrm{mV} / \mathrm{s}$ do a contribuição aniônica. Esta participação majoritária de $\mathrm{Li}^{+}$foi verificada a partir de EQCM in situ com voltametria cíclica. Considerando os princípios de conservação de massa e eletroneutralidade durante o processo redox, foi possível determinar o fluxo de elétrons, cátions e solvente + ânions (Figura 13). Assim, uma vez que a composição de um pequeno volume de eletrólito não é modificada, servindo apenas como um meio para o transporte de $\mathrm{Li}^{+}$, esses cátodos podem ser utilizados em baterias secundárias de alta energia. Além disso, a expansão/contração da estrutura é minimizada em conseqüência da mínima inserção/deinserção de solvente, melhorando a ciclabilidade do eletrodo.

Voltamogramas de sistemas híbridos formados a partir da polimerização do dímero de dissulfito 2,5-dimercapto-1,3,4-tiadiazol (diDMcT) dentro da estutura de $\mathrm{V}_{2} \mathrm{O}_{5}$ demonstraram um aumento da carga em relação ao óxido. Tal aumento foi atribuído à redução e oxidação do polímero orgânico, PDTT. Contudo, depois de vários ciclos, o perfil do voltamograma se assemelha ao $\mathrm{V}_{2} \mathrm{O}_{5}$, sugerindo que as moléculas de DMcT produzidas durante a redução dos oligômeros difundem para fora da matriz hospedeira. Este fato foi atribuído à interação repulsiva entre a rede do óxido e as espécies monotiolado e ditiolado do DMcT, carregadas negativamente em potenciais catódicos ${ }^{199}$. Na Figura 14 são mostrados o primeiro e segundo ciclos do nanocompósito PDTT/ $\mathrm{V}_{2} \mathrm{O}_{5}$, indicando a tendência na diminuição da carga atribuída a deintercalação de DMcT/PDTT.

PANI foi encapsulada dentro de $\mathrm{MoO}_{3}$ a partir da polimerização da anilina oxidando-a quimicamente através de $\left(\mathrm{NH}_{4}\right)_{2} \mathrm{~S}_{2} \mathrm{O}_{8}{ }^{200} \mathrm{e}$ $\mathrm{FeCl}_{3}{ }^{201}$. Este óxido de metal de transição, apesar de permitir a intercalação de $\mathrm{Li}^{+}$na sua estrutura, consiste em outro exemplo da baixa ciclabilidade. Nos primeiros cinco ciclos, a capacidade da cela diminui de forma considerável, em torno de $30 \%{ }^{193}$. Enquanto isso, $[\mathrm{PANI}]_{0,24} \mathrm{MoO}_{3}$ apresentou uma capacidade de $149 \mathrm{~A} \mathrm{~h} \mathrm{~kg}^{-1}$ nos dois primeiros ciclos de carga e descarga, superior a do óxido, a qual foi de $130 \mathrm{~A} \mathrm{~h} \mathrm{~kg}^{-1}$. Além disso, a eficiência coulômbica do nanocompósito foi de aproximadamente $95 \%$ nos primeiros cinco ciclos de carga e descarga, mantendo uma capacidade específica próxima à inicial.

Recentemente, nanocompósitos sintetizados a partir de um processo de secagem super crítica, denominados aerogéis, são uma nova alternativa na produção de cátodos para baterias secundárias de lítio. Curvas de carga/descarga foram realizadas para os aerogéis de nanocompósitos formados de $[\mathrm{PPy}]_{0.3} \mathrm{~V}_{2} \mathrm{O}_{5}$, a uma densidade de corrente de $10 \mu \mathrm{A} / \mathrm{cm}^{2}$ entre $3,8 \mathrm{~V}$ e $1,2 \mathrm{~V}$ (versus $\mathrm{Li}$ ). Depois do tratamento oxidativo à base de oxigênio molecular, foi observada uma intercalação de 3,8 mols de $\mathrm{Li}^{+}$para um mol do material catódico,

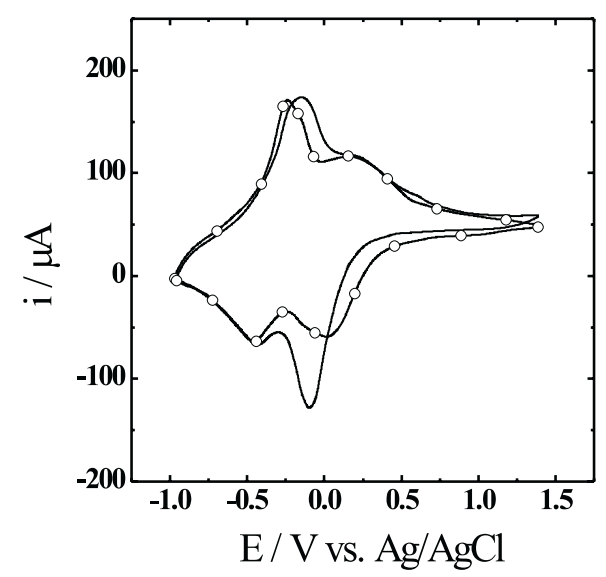

Figura 14. Voltamograma cíclico de um filme fino de PDTT- $V_{2} \mathrm{O}_{5}$ sobre Au (Primeira varredura (-); segunda varredura (-O-O-)) em $0.2 \mathrm{M}$ de $\mathrm{LiClO}_{4}$ em ACN, v $=5 \mathrm{mV} / \mathrm{s}$. Figura adaptada da ref. [199] 
mostrando um aumento superior a $40 \%$ na capacidade específica em relação ao aerogel $\mathrm{V}_{2} \mathrm{O}_{5}{ }^{202}$.

Outro exemplo da classe dos materiais híbridos é o que abrange os cátodos formados entre PCEs e os polioxometalatos como o fósforomolibdato, por exemplo ${ }^{203}$. Nesse sentido, Otero et al. ${ }^{204}$ discutiram as propriedades do compósito entre polipirrol e $\mathrm{PW}_{12} \mathrm{O}_{40}{ }^{3-}$, apesar dos problemas de dissolução do cátodo com o decorrer dos ciclos de carga/descarga, o material apresentou uma capacidade es-

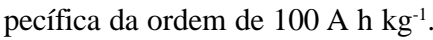

\section{PERSPECTIVAS}

As baterias secundárias de íon-Li vêm sendo amplamente estudadas no sentido de se melhorar as aplicações dessa classe de dispositivos em veículos elétricos (por exemplo o trabalho da Lithium Battery Energy Storage Technology Research Association, LIBES ${ }^{205,206}$, envolvida desde 1992 no scale-up de dispositivos secundários de íonLi). A diminuição no tamanho de aparelhos como telefones celulares, laptops, etc, torna necessário o constante aprimoramento de baterias em termos de se obter uma maior quantidade de armazenamento de carga e um maior ciclo de vida por quantidade de massa. Um fato importante a ser destacado no desenvolvimento de materiais catódicos, é a multidisciplinaridade e interdisciplinaridade dessa área de pesquisa interagindo químicos, físicos e cientistas de materiais.

A Figura 15 mostra a diferença de potencial de diferentes materias em relação a $\mathrm{Li} / \mathrm{Li}^{+}$, onde todos os materiais possuem um potencial entre 3 e $4 \mathrm{~V}$. Entretanto, o problema principal reside na capacidade de carga que é relativamente baixa fazendo com que as baterias comerciais tenham uma duração máxima entre três e quatro horas. $\mathrm{O}$ principal objetivo para o futuro, é desenvolver baterias com uma duração de pelo menos $15 \mathrm{~h}$ (como por exemplo a aplicação em livros digitais). Assim, o esforço está direcionado principalmente na obtenção de materiais com uma alta capacidade de carga. Neste ponto, aparece a utilização dos materiais clássicos, mas na forma de nanoestruturas.

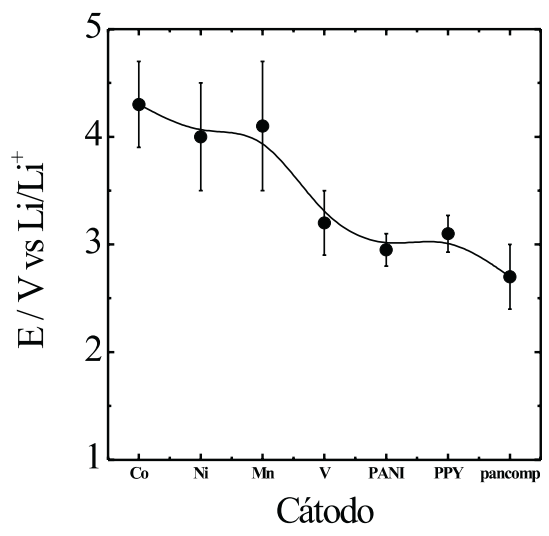

Figura15. Potencial vs $\mathrm{Li}_{/} \mathrm{Li}^{+}$de diferentes materiais utilizados como cátodos de baterias de lítio

Outro aspecto importante é a reciclagem das baterias. Em geral, todos os novos dispositivos são comparados com as baterias de chumbo-ácido, onde no momento, $90 \%$ do material destas baterias é reciclado. Portanto, além do interesse no desenvolvimento de novos materiais, são importantes também as pesquisas relacionadas com a reciclagem das baterias, tendo em vista a legislação ambiental brasileira que obriga a deposição adequada de resíduos de baterias e a utilização de compostos não agressivos ao meio ambiente.

\section{AGRADECIMENTOS}

H. Varela, F. Huguenin e M. Malta agradecem à FAPESP pelas bolsas concedidas, processos 98/00423-7, 98/15848-3 e 00/001459 , respectivamente. Também agradecemos à FAPESP e ao CNPq pelos auxílios de pesquisa.

\section{REFERÊNCIAS}

1. Brodd, R. J.; Interface 1999, 8, 20.

2. Córdoba de Torresi, S. I.; Electrochim. Acta 1995, 40, 1101.

3. Furukawa, N.; J. Power Sources 1994, 51, 45.

4. Kozawa, A.; Powers, R. A.; J. Electrochem. Soc. 1966, 113, 870.

5. Kordesch, K.; Weissenbacher, W.; J. Power Sources 1994, 51, 61.

6. Lee, J.; Varela, H.; Uhm, S.; Tak, Y.; Electrochem. Commun. 2000, 2, 646.

7. Couper, A. M.; Pletcher, D.; Walsh, F.C.; Chem. Rev. 1990, 90, 847.

8. Winter, M.; Besenhard, J. O.; Spahr, M. E.; Novak, P.; Adv. Mater. 1998, 10,725 .

9. Whittingham, M. S.; J. Electrochem. Soc. 1975, 123, 315.

10. Trumbore, F. A.; J. Power Sources 1989, 26, 65.

11. Basu, S.; Trumbore, F. A.; J. Electrochem. Soc. 1992, 139, 3379.

12. Changzhi, L.; Borong, Z.; Baochen, W.; J. Power Sources 1992, 39, 259.

13. Ein-Eli, Y.; Mcdevitt, S. F.; Aurbach, D.; Markovsky, B.; Schechter, A.; J. Electrochem. Soc. 1997, 144, L180.

14. Hayashi, K.; Nemoto, Y.; Tobishima, S.; Yamaki, J.; Electrochim. Acta 1999, 44, 2337.

15. Tarascon, J. M.; Guyomard, D.; Solid State Ionics 1994, 69, 293.

16. Guyomard, D.; Tarascon, J. M.; J. Power Sources 1995, 54, 92.

17. Dahn, J. R.; Sleigh, A. K.; Shi, H.; Way, B. M.; Weydanz, W. J.; Reimers, J. N.; Zhond Q.; Sacken, U. V. Em Lithium Batteries; Pistoia, G., ed.; Elsevier Science: Amsterdam,1994, p. 1.

18. Endo, M.; Kim, C.; Nishimura, K.; Fujino, T.; Miyashita, K.; Carbon 2000, 38, 183.

19. Dahn, J. R.; Von Sacken, V.; Juzkow, M. W.; Al-Janaby, H.; J. Electrochem. Soc. 1991, 138, 2207.

20. Aurbach, D.; Daroux, M. L.; Faguy, P. W.; Yeager, E.; J. Electrochem. Soc. 1987, 134, 1611.

21. Besenhard, J. O.; Yang, J.; Winter, M.; J. Power. Sources 1997, 68, 87.

22. Di Pietro, B.; Patriarca, M.; Scrosati, B.; J. Power Sources 1982, 2, 289.

23. Armand, M. B. Em Materials for Advanced Batteries; Murphy, D. W.; Broadhead, J.; Steele, B. C. H., ed.; Plenum: New York, 1980, p.145.

24. Nakamura, T.; Tozawa, K.; Prog. Batt. Solar Cells 1990, 9, 209.

25. Di Pietro, B.; Patriarca, M.; Scrosati, B.; J. Power Sources 1982, 8, 289.

26. Bittihn, R.; Herr, R.; Hoge, D.; J. Power Sources 1993, 43, 223.

27. Brandt, K.; J. Power Sources 1995, 54, 151.

28. Osaka, T.; Interface 1999, 8, 9 .

29. Broussely, M.; Biensan, P.; Simon, B.; Electrochim. Acta 1999, 45, 3

30. Schoonman, J.; Tuller, H. L.; Kelder, E. M.; J. Power Sources 1999, 81, 44.

31. Hossain, S. Em Handbook of Batteries; Linden, D., ed.; MacGraw-Hill: San Diego, 1994, p. 36.1.

32. Megahed, S.; Scrosati, B.; J. Power Sources 1994, 51, 79

33. Reimers, J. N.; Dahn, J. R.; J. Electrochem. Soc. 1992, 139, 2091.

34. Montoro, L. A.; Abbate, M.; Rosolen, J. M.; Electrochem. Solid-State Lett. 2000, 3, 410.

35. Plichta, E.; Slane, S.; Uchiyama, M.; Salomon, M.; Chua, D.; Ebner, W. B.; Lin, H. W.; J. Electrochem. Soc. 1989, 136, 1865.

36. Peng, Z. S.; Wan, C. R.; Jiang, C. Y.; J. Power Sources 1998, 72, 215.

37. Larcher, D.; Palacín, M. R.; Amatucci, G. G.; Tarascon, J. -M.; J. Electrochem. Soc. 1989, 136, 1865.

38. Broussely, M.; Perton, F.; Biensan, P.; Bodet, J. M.; Labat, J.; Lecerf, A.; Delmas, C.; Rougier, A.; Pérès, J. P.; J. Power Sources 1995, 54, 109.

39. Chang, C. -C.; Kumta, P. N.; J. Power Sources 1998, 75, 44.

40. Delmas, C.; Ménétrier, M.; Croguennec, L.; Saadoune, I.; Rougier, A.; Pouillerie, C.; Prado, G.; Grüne, M.; Fournès, L.; Electrochim. Acta 1999, 45, 243.

41. Broussely, M.; Biensan, P.; Simon, B.; Electrochim. Acta 1999, 45, 3.

42. Amatucci, G. G.; Tarascon, J. M.; Klein, L. C.; J. Electrochem. Soc. 1996, 143,1114

43. Delmas, C.; Saadoune, I.; Solid State Ionics 1992, 53, 370.

44. Montoro, L. A.; Abbate, M.; Almeida, E. C.; Chem. Phys. Lett. 1999, 309, 14.

45. Montoro, L. A.; Abbate, M.; Rosolen, J. M.; J. Electrochem. Soc. 2000, $147,1651$.

46. Okada, M.; Lee, Y. S.; Yoshio, M.; J. Power Sources 2000, 90, 196 
47. Lee, J. H.; Hong, J. K.; Jang, D. H.; Sun, Y. K.; Oh, S. M.; J. Power Sources 2000, 89, 7.

48. Laurindo, E. A.; Amaral, F. A.; Santos, M. L.; Ferracin, L. C.; Carubelli, A.; Bocchi, N.; Rocha-Filho, R. C.; Quim. Nova 1999, 22, 600.

49. Ferracin, L. C.; Amaral, F. A.; Bocchi, N.; Solid State Ionics 2000, 130, 215.

50. Gummow, R. J.; Thackeray, M. M.; J. Electrochem. Soc. 1998, 145, 706.

51. Teixeira, M. F. de S.; Fatibello-Filho, O.; Ferracin, L. C.; Rocha-Filho, R. C.; Bocchi, N.; Sens. Actuators, B 2000, 67, 96.

52. Thackeray, M. M.; J. Electrochem. Soc. 1997, 144, L100.

53. Kim, J.; Manthiram, A.; Electrochem. Solid-State Lett. 1999, 1, 207.

54. Koksbang, R.; Barker, J.; Shi, H.; Saïdi, M. Y.; Solid State Ionics 1996, $84,1$.

55. Xia, Y.; Zhou, Y.; Yoshio, M.; J. Electrochem. Soc. 1997, 144, 2593.

56. Blyr, A.; Sigala, C.; Amatucci, G.; Guyomard, D.; Chabre, Y.; Tarascon, J. M.; J. Electrochem. Soc. 1998, 145, 194.

57. Pasquier, A. D.; Blyr, A.; Courjal, P.; Larcher, D.; Amatucci, G.; Gèrand, B.; Tarascon, J. M.; J. Electrochem. Soc. 1999, 146, 428.

58. Tarascon, J. M.; Coowar, F.; Amatucci, G.; Schokoohi, F. K.; Guyomard, D. G.; J. Power Sources 1995, 54, 103.

59. Ohzuku, T.; Kato, J.; Sawai, K.; Hirai, T.; J. Electrochem. Soc. 1991, 138, 2556.

60. Barboux, T.; Tarascon, J. M.; Schokoohi, F. K.; J. Solid State Chem. 1991, 94, 185.

61. Armstrong, A. R.; Bruce, P. G.; Nature 1996, 381, 499.

62. Gilmor, A; J. Power Sources 1997, 65, 241.

63. Ritchie, A. G.; Giwa, C. O.; Lee, J. C.; Bowles, P.; Gilmor, A.; Allan, J.; Rice, D. A.; Brady, F.; Tsang, S. C. E.; J. Power Sources 1999, 80, 98.

64. Bruce, P. G.; Armstrong, A. R.; Gitzendanner, R. L.; J. Mater. Chem. 1999, 9, 193.

65. Bruce, P. G.; Chem. Cummun. 1997, 1817.

66. Huang, H. T.; Bruce, P. G.; J. Power Sources 1995, 54, 52.

67. Armstrong, A. R.; Gitzendanner, R.; Robertson, A. D.; Bruce, P. G.; Chem. Commun. 1998, 1833

68. Robertson, A. D.; Armstrong, A. R.; Bruce, P. G.; Chem. Commun. 2000, 1997.

69. Julien, C; Nazri, G. A. Em Handbook of Advance Electronic and Photonic Materials and Devices; Nalwa, H. S., ed.; Academic Press: San Diego, 2001, 10, p. 99.

70. Pereira-Ramos, J. P.; Baffier, N.; Pistoia, G. Em Lithium Batteries - New Materials, Developments and Perspectives; Pistoia, G., ed.; Elsevier: Amsterdam, 1994, p. 281.

71. Anaissi, F. J.; Demets, G. J. -F.; Toma, H. E.; Coelho, A. C. V.; J. Electroanal. Chem. 1999, 464, 48.

72. Lourenço, A.; Gorenstein, A.; Passerini, S.; Smyrl, W. H.; Fantini, M. C. A.; Tabacniks, M. H.; J. Electrochem. Soc. 1998, 145, 706.

73. Cruz, A. M. A.; Eon, J. G.; Appl. Catal., A 1998, 167, 203.

74. Pries de Oliveira, P. G.; Lefebvre, F.; Eon, J. G.; Volta, J. C.; J Chem. Soc., Chem. Commun. 1990, 1480.

75. Cazzanelli, E.; Mariotto, G.; Passerini, S.; Smyrl, W. H.; Gorenstein, A.; Sol. Energy Mater. Sol. Cells 1999, 56, 249.

76. Julien, C.; Ivanov, I.; Gorenstein, A.; Mater. Sci. Eng., B 1995, 33, 168.

77. Picardi, G.; Varsano, F.; Decker, F., Opara-Krasovec, U.; Surca, A.; Orel, B.; Electrochim. Acta 1999, 44, 3157.

78. Surca, A.; Orel, B.; Krasovec, U. O.; Stangar, U. L.; Drazic, G.; J. Electrochem. Soc. 2000, 147, 2358.

79. Livage, J.; Henry, M.; Sanches, C.; Progr. Solid St. Chem. 1998, 18, 259.

80. Le, D.B.; Passerini, S.; Tipton, A. L.; Owens, B. B.; Smyrl, W. H.; J. Electrochem. Soc., 1995, 142, L102.

81. Shembel, E.; Apostolova, R.; Nagirny, V.; Aurbach, D.; Markovsky, B.; J. Power Sources 1999, 81-82, 480.

82. Daí, J.; Li, S. F. Y.; Gao, Z.; Siow, K.S.; J. Power Sources 1998, 74, 40

83. Owens, B. B.; Passerini, S.; Smyrl, W. H.; Electrochim. Acta 1999, 45, 215.

84. Passerini, S.; Smyrl, W. H.; Berrettoni, M.; Tossici, R.; Rosolen, M.; Marassi, R.; Decker, F.; Solid State Ionics 1996, 90,5.

85. Gill, B. C.; Shackle, D. R.; Andersen, T. N.; J. Electrochem. Soc. 2000, 147,3575 .

86. M. E. Spahr, P. Novak, W. Scheifele, O. Haas, R. Nesper, J. Electrochem. Soc. 1998, 145, 1113.

87. Patrissi, C. J.; Martin, C. R.;J. Electrochem. Soc. 1999, 146, 3176.

88. Spahr, M. E.; Bitterli, P. S-.; Nesper, R.; Haas, O.; Novák, P.; J. Electrochem. Soc. 1999, 146, 2780.

89. Shirakawa, H.; Louis, E. J.; McDiarmid, A. G.; Chiang, C. K.; Heeger, J. A.; J. Chem. Soc., Chem. Commun. 1977, 578.

90. Panero, S.; Pila, E.; Scrosati, B.; J. Electrochem. Soc. 1996, 143, L29.

91. Scrosati, B.; Mater. Sci. Forum 1989, 42, 207.

92. Killian, J. G.; Coffey, B.; Gao, F.; Poehler, T. O.; Searson, P. C.; J. Electrochem. Soc. 1996, 143, 936
93. Coffey, B.; Madsen, P. V.; Poehlere, T. O.; Searson, P. C.; J. Electrochem. Soc. 1995, 142, 321.

94. Momma, T.; Kakuda, S.; Yarimizu, H.; Osaka, T.; J. Electrochem. Soc. 1995, 142, 1766.

95. Osaka, T.; Momma, T.; Electrochim. Acta 1993, 38, 2011.

96. Momma, T.; Nishimura, K.; Osaka, T.; Kondo, N.; Nakamura, S.; J. Electrochem. Soc. 1994, 141, 2326.

97. Furukawa, N.; Nishio, K. Em Applications of Electroactive Polymers, Scrosati, B., ed.; Chapman \& Hall: London 1993, p. 150.

98. Novák, P.; Müler, K.; Santhanam, K. S. V.; Haas, O.; Chem. Rev. 1997, 97, 207

99. Mattoso, L. H. C.; Quim. Nova 1996, 19, 388

100 .Cordoba-Torresi, S. I.; Gabrielli C.; Keddam, M.; Torresi, R.; J. Electroanal. Chem. 1990, 290, 269.

101. Barbero, C.; Miras, M. C.; Schnyder, B.; Haas, O.; Kötz, R.; J. Mater. Chem. 1994, 4, 1775.

102. Yue, J.; Epstein, A. J.; J. Am. Chem. Soc. 1990, 112, 2800

103. Wei, X. L.; Wang, Y. Z.; Long, S. M.; Bobeczko, C.; Epstein, A. J.; J. Am. Chem. Soc. 1996, 118, 2425.

104. Chen, S. A.; Hwang, G. W.; J. Am. Chem. Soc. 1994, 116, 7939.

105. Chen, S. A.; Hwang, G. W.; J. Am. Chem. Soc. 1995, 117, 10055

106. Malinauskas, A.; Holze, R.; Electrochim. Acta 1998, 43, 515.

107. Mello, R. M. Q.; Torresi, R. M.; Córdoba de Torresi, S. I.; Ticianelli, E. A.; Langmuir 2000, 16, 7835 .

108. Varela, H.; Albuqeurque Maranhão, S. L.; Mello, R. M. Q.; Ticianelli, E. A.; Torresi, R. M.; Synth. Met. 2001, 122, 321.

109. Chen S. A.; Hwang, G. W.; Macromolecules 1996, 29, 3950

110. Chen, S. A.; Hwang, G. W.; Polymer 1997, 38, 3333.

111. Varela, H.; Torresi, R. M.; Buttry, D. A.; J. Braz. Chem. Soc. 2000, 11, 32.

112. Varela, H.; Malta, M.; Torresi, R. M.; Quim. Nova 2000, 23, 664.

113. Varela, H.; Torresi, R. M.; J. Electrochem. Soc. 2000, 147, 665.

114. Varela, H., Torresi, R. M.; Buttry, D. A.; J. Electrochem. Soc. 2000, 147, 4217.

115. Gazotti Jr., W. A.; Faez, R.; De Paoli, M. A.; J. Electroanal. Chem. 1996, $415,107$.

116. Gazotti Jr., W. A.; Jannini, M. J. D. M.; Córdoba de Torresi, S. I.; De Paoli, M. A.; J. Electroanal. Chem. 1997, 440, 193.

117. Strounina, E. V.; Kane-Maguire, L. A. P.; Wallace, G. C.; Synth. Met. 1999, $106,129$.

118. Lin, H. K.; Chen, S. A.; Macromolecules, 2000, 33, 8117.

119. Hwa, M. Y.; Su, Y. N.; Chen, S. A.; Polymer 2000, 41, 813.

120. Hwang, G. W.; Wu, K. Y.; Hua, M. Y.; Lee, H. T.; Chen, S. A.; Synth. Met. 1998, 92, 39.

121. Prevost, V.; Petit, A.; Pla, F.; Synth. Met. 1999, 104, 79.

122. Ito, S.; Murata, K.; Teshima, S.; Aizawa, R.; Asako, Y.; Takahashi, K.; Hoffmann, B. M.; Synth. Met. 1998, 96, 161.

123. Inzelt, G. Em Electroanalytical Chemistry; Bard, A. J., ed.; Marcel Dekker: New York, 1994, 18, p. 89.

124. Scrosati, B.; Panero, S.; Prosperi, P.; Coraradini, A.; Mastrogostino, M.; J. Power Sources 1987, 19, 27.

125. Scrosati, B.; J. Electrochem. Soc. 1989, 136, 2774.

126. Peres, R. C. D.; Pernaut, J. M.; De Paoli, M. A.; Synth. Met. 1989, 28, C59.

127. Peres, R. C. D.; De Paoli, M. A.; Torresi, R. M.; Synth. Met. 1992, 48, 259.

128. Torresi, R. M.; Córdoba de Torresi, S. I.; Matencio, T.; De Paoli, M. A.; Synth. Met. 1995, 72, 283.

129. Varela, H.; Malta, M.; Torresi, R. M.; J. Power Sources 2001, 92, 50.

130. Kamlet, M. J.; Abboud, J. M. L.; Abraham, M. H.; Taft, R. W.; J. Org.Chem. 1983, 48,2877

131. Otero, T. F.; Cantero, I.; Grande, H.; Electrochim. Acta 1999, 44, 2053.

132. Marcus, Y.; Chem. Soc. Rev. 1993, 23, 409

133. Morita, M.; Miyazaki, S.; Tanoue, H.; Ishikawa, M.; Matsuda, Y.; J. Electrochem. Soc. 1994, 141,1409.

134. Momma, T.; Nishimura, K.; Osaka, T.; Kondo, N.; Nakamura, S.; J. Electrochem. Soc. 1994, 141, 2326.

135. Coffey, B.; Madsen, P. V.; Poehler, T. O.; Searson, P. C.; J. Electrochem. Soc. 1995, 142, 321 .

136. Varela, H; Dissertação de Mestrado, Universidade de São Paulo, Brasil, 2000.

137. Paganin, V. A.; Ticianelli, E. A.; Gonzalez, E. R.; J. Appl. Electrochem. 1996, 26, 297

138. Paganin, V. A.; Ticianelli, E. A.; Gonzalez, E. R.; J. Power Sources 1998, $70,55$.

139. Paganin, V. A.; Freire, T. J. P.; Ticianelli, E. A.; Gonzalez, E. R.; Rev. Sci. Instrum. 1997, 68, 3540.

140. Sheng, Y. L.; Quiang, S. Z.; Min, H. P.; Xiang, C. W.; Li, L.; J. Power Sources 1993, 44, 499. 
141. Matsunaga, T.; Daifuku, H.; Nakajima, T.; Kawagoe, T.; Polymers for Advanced Technologies; VCH Publishers Inc.: New York, 1990, 1, p. 33.

142. Morita, M.; Miyazaki, S.; Ishikawa, M.; Matsuda, Y.; Tajima, H.; Adachi, K.; Anan, F.; J. Electrochem. Soc. 1995, 142, L3.

143. Kolthoff, I. M.; Barnum, C.; J. Am. Chem. Soc. 1941, 63, 520.

144. Koryta, J.; Pradac, J.; J. Electroanal. Chem. 1968, 17, 177.

145. Liu, M.; Visco, S. J.; Jonghe, L. C.; J. Electrochem. Soc. 1991 138, 1891.

146. Liu, M.; Visco, S. J.; De Jonghe, L. C.; J. Electrochem. Soc. 1989, 136, 2570.

147. Liu, M.; Visco, S. J.; De Jonghe, L. C.; J. Electrochem. Soc. 1990, 137, 750 .

148. Tatsuma, T.; Yokoyama, Y.; Buttry, D. A.; Oyama, N.; J. Phys. Chem., B 1997, 101, 7556.

149. Oyama, N.; Tatsuma, T.; Sato, T.; Sotomura, T.; Nature 1995, 373, 598.

150. Liu, M.; Visco, S. J.; De Jonghe, L. C.; J. Electrochem. Soc. 1991, 138 1891.

151. Kaminaga, A.; Tatsuma, T.; Sotomura, T.; Oyama, N.; J. Electrochem. Soc. 1995, 142, L47.

152. Shouji, E.; Oyama, N.; J. Electroanal. Chem. 1996, 410, 229.

153. Pope, J. M.; Sato, T.; Shouji, E.; Buttry, D. A.; Sotomura, T.; Oyama, N.; J. Power Sources 1997, 68, 739.

154. Shouji, E.; Matsui, H.; Oyama, N.; J. Electroanal. Chem. 1997, 147, 17.

155. Shouji, E.; Buttry, D. A.; J. Phys. Chem., B 1998, 102, 1445.

156. Chi, Q.; Tatsuma, T.; Ozaki, M.; Sotomura, T.; Oyama, N.; J. Electrochem. Soc. 1998, 145, 2369.

157. Shouji, E.; Buttry, D. A.; J. Phys. Chem., B 1999, 103, 2239.

158. Bisnas, M.; Ray, S. S.; Liu, Y.; Sinth. Met. 1999, 105, 99.

159. Lira-Cantú, M.; Gómez-Romero, P.; J. Solid State Chem. 1999, 147, 601.

160. Kanatzidis, M. G.; Wu, C. G.; Marcy, H. O.; Kannzwurf, C. R.; J. Am. Chem. Soc. 1989, 111, 4139.

161. Kerr, T. A.; Wu, H.; Nazar, L. F.; Chem. Mater. 1996, 8, 2005.

162. Guinier, A.; A estrutura da matéria; EDUSP: São Paulo, 1996, p. 293.

163. Potiron, E.; Le Gal La Salle, A.; Verbaere, A.; Piffard, Y.; Guyomard, D.; Electrochim. Acta 1999, 45, 197.

164. Livage, J.; Henry, M.; Sanchez, C.; Prog. Solid St. Chem. 1988, 18, 259.

165. Pereira-Ramos, J. P.; J. Power Sources 1995, 54, 120.

166. Park, H.-K.; Smyrl, W. H.; Ward, M. D.; J. Electrochem. Soc.1995, 142, 1068.

167. Anaissi, F. J.; Demets, G. J. F.; Toma, H. E.; Electrochem. Commun. 1999, $1,332$.

168. Pistoia, G.; Antonini, A.; Zane, D.; Pasquali, M.; J. Power Sources 1995 $56,37$.

169. Johnson, C. S.; Thackeray, M. M.; Nipko, J. C.; Loong, C. K.; Physica B, 1998, 241-243, 1252.

170. Imanishi, N.; Toyoda, M.; Takeda, Y.; Yamoto, O.; Solid States Ionics 1992, $58,333$.

171. Deiss, E.; Wokaun, A.; Barra, J.-L.; Daul, C.; Dufek, P.; J. Electrochem. Soc., 1997, 144, 3877.

172. Takehara, Z.; Kanamura, K.; Yuasa, K.; Imanishi, N.; J. Electrochem. Soc. 1987, 134, c405.

173. Besenhard, J. O.; Heydecke, Fritz, H. P.; Solid State Ionics 1982, 6, 215.

174. Maia, D. J.; Alves, O. L.; De Paoli, M. A.; Synth. Met. 1997, 90, 37.

175. R. Schöllhorn, Chem. Mater. 1996, 8, 1747.

176. Wypych, F.; Seefeld, N.; Denicoló, I.; Quim. Nova 1997, 20, 356.
177. Kanatzidis, M. G.; Tonge L. M.; Marks, T. J.; J. Am. Chem. Soc. 1987, 109, 3797.

178. Kanatzidis, M. G.; Hubbard, M.; Tonge, L. M.; Marks, T. J.; Synth. Metals 1989, 28, C89.

179. Liu, Y. -J.; Schindler, J. L.; DeGroot, D. C.; Kannewurf, C. R.; Hirpo, W.; Kanatzidis; M. G.; Chem. Mater. 1996, 8, 525.

180. Kanatzidis, M. G.; Tonge, L. M.; Marks, T. J.; J. Am. Chem. Soc. 1987, 109, 3797.

181. Wu, C. G.; DeGroot, D. C.; Marcy, H. O.; Schindler, J. L.; Kannewurf, C. R.; Bakas T.; Papaefthymiou, V.; Hirpo, W.; Yesinowski, J. P.; Liu, Y.-J.; Kanatzidis, M. G.; J. Am. Chem. Soc. 1995, 117, 9229.

182. Maia, D. J.; De Paoli, M.-A.; Alves, O. L.; Zarbin, A. J. G.; das Neves, S.; Quim. Nova 2000, 23, 205

183. Kanamura, K.; Zhen, C.; Sakaebe, H.; Takehara Z.; J. Electrochem. Soc. 1991, 138, 331.

184. Kuwabata, S.; Kishimoto, A.; Tanaka, T.; Yoneyama, H.; J. Electrochem. Soc. 1994, 141, 10

185. Gemeay, A. H.; Nishiyama, H.; Kuwabata, S.; Yoneyama, H.; J. Electrochem. Soc. 1995, 142, 4190.

186. Koene, B. E.; Nazar, L. F.; Solid States Ionics 1996, 89, 147.

187. Oliveira, H. P.; Graeff C. F. O.; Rosolen, J. M.; Mater. Res. Bull. 1999, 34, 1891.

188. Livage, J.; Chem. Mater. 1991, 3, 578.

189. Oliveira, H. P.; Graeff, C. F. O.; Brunello, C. A.; Guerra, E. M.; J. NonCryst. Solids 2000, 273, 193.

190. Masbah, H.; Tinet, D.; Crespin, M.; Erre, R.; Setton, R.; Damme, H. V.; J. Chem. Soc., Chem. Commum. 1985, 935.

191. Savariault, J.-M.; Lafargue, D.; Parize, J.-L.; Galy, J.; J. Solid State Chem. 1992, 97, 169.

192. Wu, C.-G.; DeGroot, D. C.; Marcy, H. O.; Schindler, J. L.; Kannewurf, C. R.; Liu, Y.-J; Hirpo, W.; Kanatzidis, M. G.; Chem. Mat. 1996, 8, 1992.

193. Leroux, F.; Koene, B. E.; Nazar, L. F.; J. Electrochem. Soc. 1996, 143, L181.

194. Cantú, M. L.; Romero, P. G.; J. Electrochem. Soc., 1999, 146, 2029.

195. Leroux, F.; Goward G.; Power, W. P.; Nazar, L. F.; J. Electrochem. Soc. 1997, 144, 3886 .

196. Goward, G. R.; Leurox, F.; Nazar, L. F.; Electrochim. Acta 1998, 43, 1307.

197. Hwang, K. S.; Lee, C. W.; Yoon, T. H.; Son, Y. S.; J. Power Sources 1999, $79,225$.

198. Huguenin, F.; do Prado Gambardella, M. T.; Torresi, R. M.; Cordoba de Torresi, S. I.; Buttry, D. A.; J. Electrochem. Soc. 2000, 147, 2437.

199. Shouji, E.; Buttry, D. A.; Langmuir 1999, 15, 669.

200. Bissessur, R.; DeGroot, D. C.; Schindler, J. L.; Kannewurf, C. R.; Kanatzidis, M. G.; J. Chem. Soc., Chem. Commun. 1993, 687.

201. Kerr, T. A.; Wu, H.; Nazar, L. F.; Chem. Mater. 1996, 8, 2005.

202. Wong, H. P.; Dave, B. C.; Leroux, F.; Harreld, J.; Dunn, B.; Nazar, L. F.; J. Mater. Chem.1998, 8, 1019.

203. Gomez-Romero, P.; Lira-Cantu, M.; Adv. Mater. 1997, 9,144.

204. Otero, T. F.; Cheng, S. A.; Huerta, F.; J. Phys. Chem. B 2000, 104, 10522.

205. Aragane, A.; Matsui, K.; Andoh, H.; Suzuki, S.; Fakuda, H.; Ikeya, H.; Kibata, K.; Ishikawa, R.; J. Power Sources 1997, 68, 13.

206. Iwahori, T.; Mitsuishi, I.; Shiraga, S.; Nakajima, N.; Momose, H.; Ozaki, Y.; Taniguchi, S.; Awata, H.; Ono T.; Takeuchi; K.; Electrochim. Acta 2000, $45,1509$. 